\title{
The obesity-linked human IncRNA AATBC regulates adipocyte plasticity by stimulating mitochondrial dynamics and respiration
}

Maude Giroud ${ }^{* 1,2,3,4}$, Stefan Kotschi ${ }^{* 4}$, Yun Kwon ${ }^{1,2,3}$, Ophélia Le Thuc ${ }^{5}$, Anne Hoffmann ${ }^{6}$, Manuel Gil-Lozano ${ }^{1,2,3}$, Michael Karbiener ${ }^{7}$, Juan Carlos Higareda-Almaraz ${ }^{1,2,3}$, Sajjad Khani ${ }^{1,4}$, Daniel Tews $^{8}$, Pamela Fischer-Posovszky ${ }^{8}$, Wenfei Sun ${ }^{9}$, Hua Dong ${ }^{9}$, Adhideb Ghosh ${ }^{9}$, Christian Wolfrum $^{9}$, Martin Wabitsch ${ }^{8}$, Kirsi A. Virtanen ${ }^{10}$, Matthias Blüher ${ }^{6,11}$, Søren Nielsen ${ }^{12}$, Anja

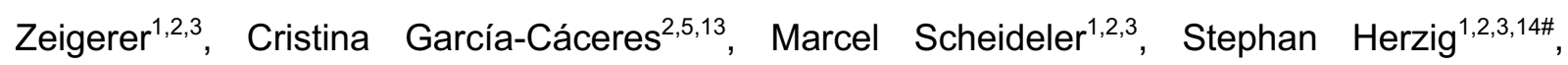
Alexander Bartelt $1,4,15,16 \#$

1 Institute for Diabetes and Cancer; Helmholtz Center Munich; 85764 Neuherberg; Germany

2 German Center for Diabetes Research; 85764 Neuherberg; Germany

3 Joint Heidelberg-IDC Translational Diabetes Program, Inner Medicine 1; Heidelberg University Hospital; 69120 Heidelberg; Germany

4 Institute for Cardiovascular Prevention, Pettenkoferstr. 9, Ludwig-Maximilians-University, 80336 Munich, Germany

5 Institute for Diabetes and Obesity; Helmholtz Center Munich; 85764 Neuherberg; Germany

6 Helmholtz Institute for Metabolic, Obesity and Vascular Research of the Helmholtz Zentrum München at the University of Leipzig and University Hospital Leipzig; 04103 Leipzig; Germany

7 Global Pathogen Safety, Baxter AG, Vienna, Austria

8 Division of Pediatric Endocrinology and Diabetes, Department of Pediatrics and Adolescent Medicine, Ulm University Medical Center, D-89075 Ulm, Germany

9 Institute of Food, Nutrition and Health, ETH Zürich, Schwerzenbach, Switzerland

10 Turku PET Centre, Turku University Hospital, Turku, 20520, Finland

11 Medical Department III - Endocrinology, Nephrology, Rheumatology, University of Leipzig Medical Center, Leipzig, Germany

12 The Centre of Inflammation and Metabolism and the Centre for Physical Activity Research, Rigshospitalet, University of Copenhagen, Copenhagen, Denmark

13 Medizinische Klinik and Poliklinik IV, Klinikum der Universität, Ludwig-Maximilians-Universität München, Munich, Germany

14 Chair Molecular Metabolic Control; Technical University Munich; 80333 Munich; Germany.

15 German Center for Cardiovascular Research, Partner Site Munich Heart Alliance, Pettenkoferstr. 9, Ludwig-Maximilians-University, 80336 Munich, Germany 
16 Department of Molecular Metabolism \& Sabri Ülker Center, 665 Huntington Avenue, Harvard T.H. Chan School of Public Health, 02115 Boston, MA, USA

*These authors contributed equally

\# Corresponding authors

stephan.herzig@helmholtz-muenchen.de

alexander.bartelt@med.uni-muenchen.de

\section{Abstract:}

Adipocytes are critical regulators of metabolism and energy balance. While white adipocyte dysfunction is a hallmark of obesity-associated disorders, the activation of thermogenic brown and beige adipocytes is linked to improved cardiometabolic health. As adipocytes dynamically adapt to environmental cues by functionally switching between white and thermogenic phenotypes, a molecular understanding of this adipocyte plasticity could help improving energy balance and weight loss. Here, we show that the long non-coding RNA (IncRNA) Apoptosis associated transcript in bladder cancer (AATBC) is a human-specific regulator of adipocyte plasticity. Searching for new human IncRNAs implicated in adipocyte biology we compared transcriptional profiles of human adipose tissues and cultured adipocytes and discovered that AATBC was enriched in thermogenic conditions. Using primary human adipocytes and immortalized human adipocytes we found that gain-of-function of AATBC enhanced the thermogenic phenotype whereas loss-of-function diminished this effect. The AATBC-mediated increase in mitochondrial respiration was linked to a more fragmented mitochondrial network and vice versa. While we found that AATBC is predominantly located in the nucleus, its effect on global transcription was only marginal. As AATBC is specific to humans, we expressed AATBC in adipose tissue of mice to study its systemic impact, which led to lower plasma leptin levels. Interestingly, this association was also present in human subjects, as AATBC in adipose tissue was inversely correlated with plasma leptin levels, body mass index and other measures of metabolic health. In conclusion, AATBC is a novel obesity-linked regulator of adipocyte plasticity and mitochondrial function in humans. 


\section{Introduction:}

Adipocytes are critical regulators of energy metabolism and nutrient homeostasis. As adipocytes are energy reservoirs and secrete endocrine hormonal regulators, they represent an important metabolic buffer system. Therefore, adipocyte dysfunction is tightly linked to metabolic disorders, including obesity, diabetes, and cardiovascular disease. While in humans, metabolic disease is mainly associated with white adipocytes, recent data indicate that thermogenic adipocytes in brown adipose tissue (BAT) play a role in energy homeostasis in humans as well [1-4]. These cells are diverse in nature and display high phenotypic plasticity [5]. Their primary function is nonshivering thermogenesis (NST), mediated by Uncoupling protein 1 (UCP1) and UCP1independent futile cycling mechanisms in response to several environmental stimuli [6-10]. The development, activity, and maintenance of thermogenic adipocytes are mainly regulated physiologically by cold-induced adrenergic stimulation. When humans are subjected to cold exposure, thermogenic adipocytes increase their metabolic activity, and if cold exposure is sustained, thermogenic adipocytes are also recruited in white adipose tissue (WAT), an adaptive phenomenon referred to as adipose tissue browning [2,11]. Conversely, when the cold stress is removed thermogenic adipocytes adopt a quiescent phenotype functionally reminiscent of white adipocytes, referred to as adipose tissue whitening [12]. In mice, thermoneutrality is ca. $30{ }^{\circ} \mathrm{C}$ [13], but humans are thermoneutral at much lower temperatures and, therefore, humans only display limited thermogenic activity [14], as their warm environment makes NST obsolete. However, in colder regions, people display markedly increased NST [2], indicating that the environmental temperature are major determinant of BAT activity. Furthermore, activating BAT has remarkable benefits for metabolic health in mouse models of metabolic disorders [15-17], but also, in the general human population BAT is linked to better cardiometabolic health [18]. Understanding the molecular switches and hormonal cues regulating thermogenic plasticity in adipocytes, thus dictating the balance of energy storage vs energy dissipation, might help to develop novel therapies for treating obesity-associated metabolic disorders.

Key aspects of thermogenic plasticity include facilitating an increase of mitochondrial respiration in response to cold, and conversely, directing the involution of mitochondrial capacity when the adipocytes return to a non-thermogenic state at thermoneutrality. In most cells, a fragmented mitochondrial network is associated with stress, whereas fused mitochondria are thought to display enhanced respiration [19]. In the case of adipocytes, mitochondrial dynamics are largely regulated by nutrient availability and depend on the thermogenic status of the adipocyte [20-23]. The interplay of molecules governing these mitochondrial adaptations are complex and involve a large panel of transcriptional and posttranslational regulators, for example peroxisome 
proliferator-activated receptor co-activator 1-alpha (PGC1 $\alpha$ ) [24], Nuclear factor erythroid-2, like1 (NFE2L1, also known as NRF1 or TCF11) [25], Mitofusin-1 and -2 (MFN1 and MFN2) [26-28], OPA1 mitochondrial dynamin like GTPase (OPA1) [21, 29] as well as dynamin 1 like (DNM1L, also known as DRP1) [23]. For example, adipocyte-specific Mfn2-deficient mice display blunted mitochondrial fusion and impaired adaptative thermogenesis [27, 28]. In human adipocytes, it has been shown previously that an acute activation of thermogenic adipocytes involves fission of the mitochondrial network for enhanced respiration [23, 26].

In addition to these transcriptional and posttranslational mechanisms, non-coding RNAs and especially long non-coding RNAs (IncRNAs) have emerged as potential regulators of adipocyte function [30-34]. The number of IncRNAs in the genome is correlated with organism complexity, they are highly tissue-specific, and their sequence is only poorly conserved between species [35]. They are found across the entire genome and adopt secondary structures that confer several regulatory properties. IncRNA are longer than 200 nucleotides and bind to many types of molecules including RNA, DNA, amino acids, and proteins [36]. In addition to their interactome, their cellular localization also defines their function. For example, when localized to the nucleus, IncRNA impact transcriptional control, genomic imprinting, and chromatin condensation [37]. Furthermore, in the cytoplasm, IncRNAs are decoys for mRNA translation and miRNA function, thus affecting mRNA turnover [38]. The study of IncRNAs in metabolism is an emerging field. In adipocytes, IncRNA have been linked to adipogenesis [34]. For example, Brown fat IncRNA 1 (BInc1) and brown adipose tissue-enriched IncRNA 10 (IncBATE10) promote the development of thermogenic adipocytes through the regulation of Early B Cell Factor 2 (EBF2) and PGC1 $\alpha$, respectively [42-44]. CCCTC-binding factor (zinc finger protein)-like, opposite strand (Ctcflos) was described as a regulator of browning via regulation of PR/SET Domain 16 (PRDM16) splicing [45]. However, most of these IncRNAs are specific to mice and are not found in humans. One study found 318 IncRNAs conserved in humans and mice [46]. To the best of our knowledge, LINC00473 is so far the only non-conserved, human IncRNA implicated in the regulation of thermogenic adipocytes[47].

In the current study, we set out to discover new IncRNAs specific to human adipocytes and linked to NST and obesity. Based on a transcriptional comparative analysis between human WAT and BAT as well as adrenergic stimulation of different models of cultured human adipocytes, we show that the IncRNA Apoptosis associated transcript in bladder cancer (AATBC) [48-51], regulates thermogenic plasticity of adipocytes by stimulating mitochondrial dynamics and respiration, and is linked to human obesity. 


\section{Material and Methods}

\section{Cell culture:}

Human SVF (RNAseq screening, figure 1):

Human primary adipose-derived stem cells (hpASCs) were isolated and stimulated as previously described [52]. In brief, subcutaneous lipoaspirates from healthy female donors $(n=4)$ were thawed, cultivated in EGM-2 Medium (Lonza), and used after 1-3 passages. 2 days after the cells reached confluency (= day 0 ), adipocyte differentiation was induced with the following medium (DMEM/Ham's F12 (50:50)) supplemented with $5 \mathrm{mM}$ HEPES, $2 \mathrm{mM} \mathrm{L-glutamine,} 100 \mu \mathrm{g} / \mathrm{ml}$ normocin, $860 \mathrm{nM}$ insulin, $10 \mu \mathrm{g} / \mathrm{ml}$ apo-transferrin, $100 \mathrm{nM}$ rosiglitazone, $0.2 \mathrm{nM}$ triiodothyronin) supplemented with $100 \mu \mathrm{M}$ 3-isobutyl-1-methylxanthine (IBMX), and $1 \mu \mathrm{M}$ dexamethasone (Dex). At day 7 of differentiation, supplementation with insulin was stopped. At day 9 of differentiation, the differentiated adipocytes were stimulated with $1 \mu \mathrm{M}$ norepinephrine (NE; dissolved in $10 \mathrm{mM}$ $\mathrm{HCl}$ ) or vehicle (VE, $10 \mathrm{mM} \mathrm{HCl}$ ) for 3 hours.

\section{hMADS cells:}

Human multipotent adipose derived stem (hMADS) cells were cultured as previously described [53]. Briefly, cells were kept until confluency in proliferation medium (Dulbecco's Modified Eagle's Medium (DMEM), (Lonza, Switzerland \# BE12-707F) supplemented with $10 \%$ FBS (Sigma, Germany), $10 \mathrm{mmol} / \mathrm{l}$ 4-(2-hydroxyethyl)-1-piperazineethanesulfonic acid (HEPES) (Gibco, Germany \#15630056), $2.5 \mathrm{ng} / \mathrm{ml}$ human fibroblast growth factor-basic (hFGF2) (PeproTech, Germany \#100-18B), $50 \mathrm{mg} / \mathrm{ml}$ penicillin, and $50 \mathrm{mg} / \mathrm{ml}$ streptomycin (Gibco, Germany \#15140122), followed by $48 \mathrm{~h}$ of incubation without hFGF2. The induction of the differentiation towards adipocytes was started (d0) using a cocktail of DMEM/Ham's F12 (Lonza, Switzerland \# BE12-615F) supplemented with $10 \mathrm{mg} / \mathrm{ml}$ apo-transferrin (Sigma, Germany \# T1428), $10 \mathrm{nM}$ insulin (Sigma, Germany \# I 9278), 100 nM rosiglitazone (Cayman, Germany \#71740), 0.2 nM triiodothyronine (T3) (Sigma, Germany \#6893-02-3), 1 mmol/l dexamethasone (DEX) (Sigma, Germany \# D4902), 1 mM 3-isobutyl-1-methylxanthine (IBMX) (Sigma, Germany \#28822-58-4). After $48 \mathrm{~h}$ the induction medium was replaced by the differentiation medium containing DMEM/Ham's F12, $10 \mathrm{mg} / \mathrm{ml}$ transferrin, $10 \mathrm{nM}$ insulin, $0.2 \mathrm{nM}$ triiodothyronine and $1 \mu \mathrm{M}$ rosiglitazone. Until day 9 , the medium was changed every other days. At day 9 , thermogenic differentiated hMADS cells were obtained with chronic rosiglitazone treatment until day of completion of the experiment (d14). 


\section{Human SVF purification and differentiation:}

Human stromal vascular fraction was purified from subcutaneous adipose tissue from patients undergoing abdominoplasty. The study was approved by the ethical committee (vote no. 300/16) of the University of UIm after written informed consent. Minced tissue was chemically digested using collagenase (Sigma, Germany \#11088793001) in BSA (Sigma, Germany \#A8806-5G) media (ratio 1:3) for 30-45 min until the digestion was stopped by the addition of FBS (Sigma, Germany) at a $10 \%$ v/v ratio. To purify primary adipocytes from of the digested tissue, the mixture was first filtered consecutively through $100 \mu \mathrm{m}, 70 \mu \mathrm{m}$ and $48 \mu \mathrm{m}$ meshes and then centrifugated to finally separate the primary adipocytes in the pellet from the supernatant. Cells were plated and treated like hMADS cells.

Human SVF purification and differentiation (refers to figures $2 A$ and $2 B$ ):

Preadipocytes isolated from the supraclavicular and abdominal subcutaneous region were cultured, differentiated and stimulated as previously described [54]. RNA was extracted from adipocytes using the Trizol method. RNA sequencing was performed by BGI (Hong Kong) using 1000 ng RNA for the TruSeq cDNA library construction (Illumina). 3Gb data was generated per sample on a HiSeq 2000 sequencer (Illumina) and a 91-paired end sequencing strategy was used. Read quality was assessed using FastQC (http://www.bioinformatics.babraham.ac.uk) and the following pre-processing steps where performed using the Fastx toolkit (http://hannonlab.cshl.edu) and PRINSEQ: $7 \mathrm{nt}$ were clipped off from the 5'-end of every read [55]. The reads were then filtered to remove all $\mathrm{N}$-reads. The $3^{\prime}$-ends were then trimmed, and the reads filtered to minimum Q25 and 50 bp length. Reads were then mapped with tophat2 to the human genome GRCh38 Ensembl release 77 . Read counts were imported into R, and DESeq2 was used for identifying differential expression.

\section{Modulation of gene expression:}

The expression of $A A T B C$ was modulated in vitro using antisense oligonucleotide-mediated knockdown (ASO, Exiqon, \#634048-6, \#634048-4), siRNA-mediated knockdown (Lincode Human AATBC (Horizon Discovery, \#284837) and adenovirus (AV) mediated overexpression (VectorBuilder, \#AVM (VB150925-10024) + GFP). White hMADS were transfected at day 12 of differentiation with $30 \mathrm{nmol}$ of siRNA or ASO at day 10 using Lipofectamine ${ }^{\mathrm{TM}}$ RNAiMAX transfection reagent (Thermo Scientific, \#13778075) according to the manufacturer's protocol. For overexpression, white hMADS cells were infected with 200 particles of virus per cell. $24 \mathrm{~h}$ 
later, the transfection medium was removed and replaced by the suitable differentiation medium. $48 \mathrm{~h}$ after transfection cells were harvested.

\section{Nuclear RNA extraction:}

Nuclear RNA was purified using a Cytoplasmic \& Nuclear RNA Purification Kit (Norgen, Canada \# 21000) according to the manufacturer's instructions. cDNA was synthesized with Maxima ${ }^{\mathrm{TM}} \mathrm{H}$ Master Mix 5x (Thermo Fisher Scientific, \#M1661), followed by qPCR according to the PowerUp ${ }^{\text {TM }}$ SYBR Green technology (Applied Biosystems, \#A25741)

\section{Immunoblot:}

Protein expression was investigated using western blot as described previously [53]. Cells were lysed in RIPA buffer (150 mM NaCl, $5 \mathrm{mM}$ EDTA, $50 \mathrm{mM}$ Tris $\mathrm{pH}$ 8, $0.1 \% \mathrm{w} / \mathrm{v}$ SDS, $1 \% \mathrm{w} / \mathrm{v}$ IGEPAL $®$ CA-630, $0.5 \%$ w/v sodium deoxycholate) containing protease inhibitors (Sigma) in a $1: 100 \mathrm{v} / \mathrm{v}$ ratio. Protein concentrations of the lysates were determined using Pierce BCA Protein Assay (Thermo Scientific) following the manufacturer's protocol. $30 \mu \mathrm{g}$ of total protein were loaded per well on Bolt ${ }^{\mathrm{TM}} 4-12 \%$ Bis-Tris gels (Thermo Scientific, \#NW04120BOX) and blotted on a $0.2 \mu \mathrm{m}$ PVDF membrane (Bio-Rad, \#1704156) using the Trans-Blot $\AA$ Turbo ${ }^{\mathrm{TM}}$ system. Membranes were incubated in primary antibodies at a dilution of 1:1000 v/v in ROTI-Block (Roth, Germany \#A151.1) after blocking in ROTI-Block Secondary antibodies (concentration of 1:10000 $\mathrm{v} / \mathrm{v}$ in ROTI-Block. All antibodies used are shown in Table 1. After antibody incubation membranes were washed $4 \mathrm{x}$ for $10 \mathrm{~min}$ in TBS-T (Tween $0.1 \%$ ). Bands were detected using a Chemidoc MP Sytstem (Bio-Rad, \#1704156).

\section{Gene expression analysis:}

RNA of cells and tissues were extracted using TRIzol (Invitrogen, \#15596026) and NucleoSpin® RNA kit (Macherey-Nagel, \#740955.250) according to the manufacturer's instruction. cDNA was synthesized with Maxima ${ }^{\mathrm{TM}} \mathrm{H}$ Master Mix $5 \times$ (Thermo Fisher Scientific, \#M1661), followed by qPCR according to the PowerUp ${ }^{\mathrm{TM}}$ SYBR Green technology (Applied Biosystems, \#A25741) using the primers listed in Table 2. Gene expression was calculated using the $\Delta \Delta$ Ct-method normalized to the housekeeping gene indicated in the respective figure.

\section{Analysis of mitochondrial respiration:}

To assess mitochondrial respiration a Seahorse XFe24 device (Agilent \# 102238-100, S7801A) was used. hMADS cells were seeded differentiated and transfected or transduced at day 12 as 
described above. Oxygen consumption rate was determined during a Mito Stress Test $(1 \mu \mathrm{M}$ Oligomycin-A (Sigma-Aldrich, \#75351), 1.2 $\mu$ M FCCP (Sigma-Aldrich, \#C2920), $2 \mu M$ Rotenone (Sigma-Aldrich, \#R8875), $2 \mu \mathrm{M}$ Antimycin A (Sigma-Aldrich, \#A8674)) with additional treatment with Isoproterenol (100 nM, Sigma-Aldrich, \#I5627-5G).

\section{Quantification of mitochondrial DNA:}

To assess cellular mitochondrial amount, DNA was extracted using a DNA Extraction Kit (Macherey-Nagel, \#740952.50) and used for qPCR as described before [56]. The expression of the mitochondrial gene $N A D H$ was normalized to the single copy nuclear gene $L P L$.

\section{In situ hybridization - RNAscope®:}

To visualize the intracellular localization of ATTBC, we used the RNAscope $®$ Multiplex Fluorescent Reagent Kit v2- Hs (ACD Bio, \#323135). Undifferentiated hMADS cells were seeded on coverslips in 24-well tissue culture dishes, grown to confluence and differentiated into thermogenic adipocytes for 12 days (see hMADS cells differentiation). In situ hybridization was performed according to the manufacturer's protocol (Advanced Cell Diagnostics), using a probe designed to specifically detect human AATBC (RNAscope ${ }^{\circledR}$ Probe Hs-AATBC (Advanced Cell Diagnostics, \#519681)), in combination with the Opal 620 Fluorophore Reagent (Akoya Biosciences). Cells were visualized on a Leica TCS SP5 confocal microscope (Leica, Germany). Images were acquired for all conditions as z-stacks (steps of $0.5 \mu \mathrm{m}$ along the $z$ axis) with a glycerol-based immersion fluid-immersed $63 \mathrm{x}$ objective. Acquisition parameters were kept consistent for all images. The same image processing was performed using ImageJ (FIJI) software for the different conditions.

\section{Mitochondria 3D Reconstruction and Morphometric Analysis:}

Immunofluorescence was performed at day 14 of differentiation as previously described [23]. To visualize the mitochondrial network TOMM20 antibody (Abcam, \#ab78547, 1:1000 v/v) was used followed by incubation with a fluorescent antibody (molecular probes, A-21429, 1:1000 v/v). Immunofluorescent samples were analyzed using a Laser Scanning Confocal Microscope (Olympus Fluoview 1200, Olympus, Tokyo, Japan) equipped with an Olympus UPlanSApo $60 \mathrm{x}$ 1.35 and an UplanSApo $40 \times 1.25$ Sil Oil immersion objective (Olympus, Tokyo, Japan) at a resolution of app. $100 \mu \mathrm{m} /$ pixel (60x) and $600 \mathrm{~nm}$ step size. For mitochondrial 3D reconstruction, images were deconvolved using the FIJI plugins point spread function (PSF) generator [57] and DeconvolutionLab [58]. Z-step was set to $0.6 \mu \mathrm{m}$ and a PSF algorithm (Born \& Wolf 3D Optical 
model) was used for PSF generation, as previously described [59]. The generated PSF and a 3D deconvolution algorithm (Richardson-Lucy with TV regularization) were applied to microscopic images using DeconvolutionLab. From the deconvolved 2D and 3D binary images (8-bit images), mitochondrial network was determined by generating a skeleton of the images using the Fiji plugin Skeletonize3D and analyzed using the plugin AnalyzeSkeleton (2D/3D). This plugin tags all pixel/voxels in a skeleton image and then counts the junctions and branches of the mitochondrial network and measures their average length. For mitochondrial network analysis, minimum of 20 cells were analyzed.

\section{Mouse experiments:}

Animal experiments were performed in accordance with German animal welfare legislation and approved by the state ethics committee and government of Upper Bavaria (no. ROB-55.22532.Vet_02-17-125). Mice were group-housed at $22{ }^{\circ} \mathrm{C}$ with a $12 \mathrm{~h}$ dark-light cycle in the animal facility of Helmholtz Center Munich. 9-week-old male C57BL/6J mice were purchased from Janvier Labs and acclimatized in the Helmholtz center facility for 3 weeks. Induction of anesthesia was performed using $4 \%$ isoflurane and upheld by inhalation of $2 \%$ isoflurane. Incisions were made over the intrascapular brown adipose tissue and inguinal adipose tissue. Injections of $25 \mu \mathrm{l}$ containing $4^{*} 10^{7}$ viral particles of AV_AATBC were performed into each lobe of the tissues. For glucose tolerance test (GTT), performed at day 7 and insulin tolerance test (ITT), performed at day 14 , mice were fasted for $6 \mathrm{~h}$ after which they received intraperitoneal injections of glucose ( $3 \mathrm{~g} / \mathrm{kg}$ for GTT) and insulin (0.7 U/kg for ITT). Blood glucose was measured from the tail vain at $15,30,60,90$ and 120 minutes after injection. After 12 days mice were euthanized using cervical dislocation and necropsy was performed. Plasma was analyzed for metabolic parameters including adiponectin, triglycerides and non-esterified fatty acids using a serum analyzer (AU480 Chemistry analyzer Beckman Coulter). Plasma levels of leptin and insulin were assessed using ELISAs used according to the manufacturer's instructions (R\&D Systems, \#MOB00B and Crystal Chem, \#90082, respectively).

\section{Human data:}

Human adipose tissue samples (RNAseq screening, refers to figure 1):

Human BAT and WAT were removed from the same incision site in the supraclavicular localization, BAT being fluorodeoxyglucose-positron emission tomography (FDG-PET)-positive scan sites and WAT being not. The Study protocol was approved by the ethics committee of the Hospital District of Southwestern Finland, and subjects provided written informed consent 
following the committee's instructions. The study was conducted according to the principles of the Declaration of Helsinki. All potential subjects who donated BAT were screened for metabolic status, and only those with normal glucose tolerance and normal cardiovascular status (as assessed based on electrocardiograms and measured blood pressure) were included. The age range of the subjects was $23-49$ years. We studied a group of 5 healthy volunteers ( 3 females, 2 males).

Human samples (RNAseq, refers to figures 6 and 7):

The acquisition of human data from cohorts 1 and 2 has been previously described [53]. Briefly, cohort 1 includes 318 individuals (249 female individuals, 69 male individuals; BMI range: 21.9 $97.3 \mathrm{~kg} / \mathrm{m}^{2}$, age range: $19-75$ years) undergoing elective laparoscopic surgery during which subcutaneous (scWAT) and visceral, omental (visWAT) adipose depots were obtained__Cohort 2 and 3 are from the Leipzig Obesity Biobank. Cohort 2 includes 96 individuals (23 lean female individuals, mean age $43.3 \pm 7.4$ years, mean BMI $23.7 \pm 1.3 \mathrm{~kg} / \mathrm{m}^{2}$ ), 48 obese female individuals, mean age $42.9 \pm 8.3$ years, mean BMl $\left.45.9 \pm 6.1 \mathrm{~kg} / \mathrm{m}^{2}\right), 9$ lean male individuals, mean age $44.7 \pm 7.1$ years, mean BMI $22.3 \pm 1.8 \mathrm{~kg} / \mathrm{m}^{2}$ ), and 16 obese male individuals, mean age $42.2 \pm 7.6$ years, mean BMl $\left.44.9 \pm 5.2 \mathrm{~kg} / \mathrm{m}^{2}\right)$ ). Cohort 3 comprises 1415 individuals, with visWAT samples collected from 920 individuals (female lean $(n=45$, mean age $62.1 \pm 13.7$ years, mean BMI $\left.24.4 \pm 2.9 \mathrm{~kg} / \mathrm{m}^{2}\right)$, female obese $(n=582$, mean age $46.1 \pm 11.8$ years, mean BMI $\left.48.5 \pm 8.5 \mathrm{~kg} / \mathrm{m}^{2}\right)$, male lean $(n=44$, mean age $61.4 \pm 15.7$ years, mean BMI $\left.24.9 \pm 3.5 \mathrm{~kg} / \mathrm{m}^{2}\right)$, and male obese $(n=249$, mean age $48.2 \pm 11.7$ years, mean BMI $\left.\left.49.1 \pm 8.5 \mathrm{~kg} / \mathrm{m}^{2}\right)\right)$ and scWAT samples taken from 814 individuals (female lean $(n=33$, mean age $63.8 \pm 12.9$ years, mean BMI $\left.24.7 \pm 2.8 \mathrm{~kg} / \mathrm{m}^{2}\right)$, female obese $(n=545$, mean age $46.7 \pm 12.0$ years, mean BMI $\left.48.3 \pm 9.2 \mathrm{~kg} / \mathrm{m}^{2}\right)$, male lean $(n=25$, mean age $65.6 \pm 14.3$ years, mean BMI $\left.25.5 \pm 2.6 \mathrm{~kg} / \mathrm{m}^{2}\right)$, and male obese $(n=211$, mean age $48.09 \pm 12.4$ years, mean BMI $\left.49.5 \pm 8.0 \mathrm{~kg} / \mathrm{m}^{2}\right)$ ). Patients were classified as lean when their BMI was less than $30 \mathrm{~kg} / \mathrm{m}^{2}$. For cohort 3, human single-end and rRNA-depleted RNA-seq data were prepared with a SMARTseq protocol [60,61]. In brief, RNA was enriched and reverse transcribed by Oligo(dT) and TSO primers. cDNA was amplified by ISPCR primers and processed with Tn5 using Nextera DNA Flex kit. All libraries were sequenced on an Novaseq 6000 instrument at Functional Genomics Center Zurich (FGCZ). Approval for all 3 studies was obtained from the Ethics Committee of the University of Leipzig (approval no: 159-12-21052012) before the study and acquisition was performed in accordance with the declaration of Helsinki. 


\section{Bioinformatic Analysis:}

Bioinformatic Analysis (RNAseq screening, refers to figure 1):

RNA quality was assessed using BioAnalyzer 2100 (Agilent); all samples had RIN values $\geq 8.5$. $4 \mu \mathrm{g}$ total RNA per sample were used for the TruSeq Stranded mRNA LT Sample Prep Kit (Illumina) to generate cDNA libraries according to the manufacturer's protocol. Single read sequencing was carried out using Illumina/Solexa HiSeq 2000. High-throughput sequencing was conducted by the Biomedical Sequencing Facility, Vienna. RNAseq alignment, long non-coding quantification and differential expression analysis were performed as follows: Raw sequencing reads were aligned against the human hg38 genome using STAR aligner with default parameters [62]. The mapped reads were assigned to genes using featureCount from the Bioconductor package Rsubread [63]. All annotated IncRNAs were quantified across each condition, using hg38 annotation. Normalization and differential expression analysis were performed using the R/Bioconductor package DESeq2 [64]. Significance was assumed for an adjusted $p$-value $<0.01$. Data of the hpAS treated with norepinephrine are publicly available sequencing data [36].

Bioinformatic Analysis (RNAseq human cohort 3, refers to figures 6 and 7):

Adapters and low quality bases of raw reads were trimmed using fastp v0.20.0 [65]. Only reads with a minimum read length of $18 \mathrm{nts}$ and which surpass a quality cut-off of 20 were kept. The remaining reads were aligned against the human (GRCh38.p13) genome from GENCODE [66] applying the STAR alignment algorithm v2.7.4a [67], allowing 50 multiple alignments per read. Standard pre- and post-mapping quality control was computed using FASTQC v.0.11.4 (https://www.bioinformatics.babraham.ac.uk/projects/fastqc). Gene counts were conducted with featureCounts v2.0.1 (10.1093/bioinformatics/btt656) where multiple mapped reads were fractionally counted. Count data were homoscedastic normalized with respect to library size using the variance stabilizing transformation from DESeq2 v1.32.0 [64]. Correlation analyses were computed using the psych R package v2.1.6 with the spearman correlation coefficient and confidence intervals of 0.05 . P-values were adjusted using the Holm's method (http://www.jstor.org/stable/4615733). Visualization of correlation analysis was performed with the corrplot v0.90 and ggstatsplot v0.8.0 R packages under $\mathrm{R}$ version 4.1.

\section{Statistics:}

Data is presented as mean \pm standard error of mean (s.e.m). Student's t-test and 2-way ANOVA were used as indicated in the figure legends and performed using GraphPad Prism 9.0 and $R$ 
bioRxiv preprint doi: https://doi.org/10.1101/2021.08.09.455681; this version posted August 9, 2021. The copyright holder for this preprint (which was not certified by peer review) is the author/funder. All rights reserved. No reuse allowed without permission.

4.1.0. In vitro experiments were performed at least 3 times and pooled where possible. Differences were deemed significant with a P-value $<0.05$ and indicated by an asterisk. 


\section{Results:}

\section{Identification of AATBC as a human IncRNA linked to NST in vivo and in vitro.}

Investigating the phenotypic signatures of different adipose depots in humans as well as isolated adipocytes offers the opportunity to unravel novel IncRNAs implicated in the control of thermogenic plasticity. Therefore, we performed a combined RNAseq analysis of three different human models. We compared IncRNA expression profiles of (1) WAT and BAT ex vivo, (2) differentiated human primary adipose-derived stem (hpAS) cells treated with norepinephrine (NE) and (3) human adipose derived stem (hMADS) cells treated with forskolin (Figure 1A). To confirm the induction of the thermogenic phenotype, we quantified UCP1 mRNA expression in all samples (Figure $1 \mathrm{C}$ ). Other thermogenic markers such as citrate synthase (CS), cell death inducing DFFA like effector $A(C I D E A)$, peroxisome proliferator-activated receptor gamma coactivator 1- $\alpha$ $(P P A R G C 1 A)$ and adipose markers such as peroxisome proliferator-activated receptor- $\gamma$ (PPARG), perilipin 1 (PLIN1) and fatty acid binding protein 4 (FABP4) were quantified for quality control purposes (Supplementary figure $G-X$ ). After rigorous filtering (Supplementary figure 1AD), we found 368 IncRNAs to be differentially regulated in WAT vs BAT, 105 IncRNAs in hpAS treated with NE compared to control cells, and 116 IncRNAs in hMADS stimulated with forskolin compared to control cells, respectively. Out of these differentially regulated set of IncRNAs, 25 candidates were regulated in the same fashion under all three conditions (Figure 1B). Among these, we found that the majority of IncRNAs had higher expression levels under thermogenic conditions (Figure 1F and Supplementary figure 1E,F). Interestingly, among the IncRNAs with unknown function in adipocytes, the IncRNA AATBC was one of the most prominent candidates, which was consistently upregulated in all three models of stimulated thermogenesis. Of note, we found that LNC00473 was consistently upregulated, which is in agreement with a previous study on human IncRNAs [47]. These findings indicate that our stratification strategy was valid. (Figure $1 \mathrm{~F}-\mathrm{L}$ and Supplementary figure $1 \mathrm{E}, \mathrm{F}$ ). In summary, the thermogenic phenotype of adipocytes is characterized by an induction of IncRNA transcription. In the pool of upregulated IncRNAs, AATBC is consistently associated with increased thermogenesis in human adipose tissue and cultured adipocyte models.

\section{AATBC is a nuclear IncRNA induced by thermogenic activation and differentiation.}

As AATBC matched the candidate profile for a IncRNA associated with thermogenic features, we further explored the nature and regulation of AATBC expression in our in vitro models of human adipocytes. Confirming the results of the initial RNASeq screening, AATBC expression was higher 
in primary human brown adipocytes treated with NE (Figure 2A, B). As adipocyte browning is also induced by chronic PPAR $\gamma$ activation [68], we treated hSVF with the PPAR $\gamma$ agonist rosiglitazone during the differentiation (Supplementary figure 2A-C). Also, in this distinct model of adipocyte browning AATBC expression was higher under thermogenic conditions compared to controls cells differentiated to classical white adipocytes (Figure 2C). Using adipose tissue fractionation, we found that AATBC was expressed at higher levels in differentiated adipocytes compared to SVF cells (Figure 2D). Also, in hMADS cells we observed higher levels of AATBC in the respective models of thermogenic and regular differentiation (Figure 2E-F). UCP1, PLIN1 and FABP4 expression levels served as validation markers for the thermogenic and regular differentiation, respectively (Supplementary figure 2D-K). Next to expression levels, the localization of IncRNAs is very important for understanding their function. To this end, we separated nuclear and cytoplasmic RNA from differentiated thermogenic hMADS cells (Figure 2J,K) and found that $A A T B C$ was enriched in the nuclear fraction (Figure 2l). The finding that AATBC is a nuclear IncRNA was corroborated by in situ hybridization of AATBC (RNAscope ${ }^{\circledR}$ ) in the same hMADS cells. There, endogenous expression of AATBC was detected in the nucleus. (Figure $2 \mathrm{~L}$ ). This observation was confirmed by overexpression of AATBC, after which in most cells AATBC was found in the nucleus (Figure $2 \mathrm{M}$ ). In summary, our data demonstrate that AATBC is a nuclear IncRNA and is induced by thermogenic activation and differentiation.

\section{Modulation of AATBC expression regulates mitochondrial oxygen consumption.}

To investigate the biological significance of AATBC we performed loss and gain-of-function experiments in human adipocytes. We used anti-sense oligonucleotide (ASO)-mediated knockdown of AATBC in hMADS cells under thermogenic conditions, where otherwise AATBC expression is high, and adenoviral over-expression of AATBC in regular hMADS cells, where otherwise AATBC expression is low. (Figure $3 A$ ). Using two independent ASOs we found that AATBC silencing led to lower UCP1 expression (Figure 3B, C). These observations were confirmed in primary cells derived from hSVF (Supplementary figure $3 A, B$ ). Similarly, an alternative approach using siRNA against AATBC led to similar results in hMADS cells (Supplementary figure 3E,F). As changes in UCP1 levels might indicate altered mitochondrial function, we assessed the bioenergetic profiles of hMADS with silenced AATBC and control cells. We found that silencing of AABTC diminished basal, isoproterenol-stimulated, uncoupled respiration as well as lower spare mitochondrial capacity (Figure 3D-H). Taken together, this data set indicates that loss of AATBC disrupts the thermogenic function of adipocytes. In the complementary approach, we found that after overexpression of AATBC UCP1 expression 
remained unchanged (Figure 3I,J). Yet we observed higher basal respiration and higher spare mitochondrial capacity compared to control cells. However, isoproterenol-stimulated respiration and proton leak remained unchanged, which is in line with the unchanged UCP1 expression (Figure 3K-O). Of note, PLIN1 and FABP4 expression levels were largely unchanged, indicating that manipulation of AATBC did not interfere with adipogenesis per se (Supplementary figure 3C, $D, G, H, I-L)$. Next, we investigated if the manipulation of AATBC function is linked to any transcriptional changes that could explain the mitochondrial phenotype. We silenced AATBC in thermogenic hMADS using siRNA and performed RNASeq. We found that despite a marked reduction in $A A T B C$ levels, the effect on global gene regulation was minor and only few genes were impacted by the loss of AATBC without any clear outcome (Supplementary figure 4A-C). We also performed a transcriptomic analysis of regular hMADS overexpressing AATBC and found lipid and triglyceride metabolism pathways to be regulated (Supplementary figure 4D-F). Regardless of these observations, the transcriptomic changes upon of AATBC manipulation were not immediately linked to mitochondrial function, potentially indicating the AATBC exerts its effects largely via posttranscriptional mechanisms.

\section{Modulation of AATBC expression regulates mitochondrial dynamics.}

Considering that AATBC had only minor impact on the transcriptome of thermogenic adipocytes, we hypothesized that the mitochondrial phenotype is manifested at the posttranslational level. First, we quantified mitochondria DNA, but neither inhibition nor overexpression of AATBC led to altered mitochondrial DNA levels, suggesting that the number of mitochondria was unchanged under these conditions (Supplementary figure 5A,B). Next, we investigated mitochondrial dynamics, as mitochondrial fission and fusion events regulate mitochondrial respiration [22, 69]. Using TOM20 immunostaining, we found that silencing of AATBC in thermogenic hMADS was associated with higher numbers and length of mitochondrial branches as well as more mitochondrial junctions per cell area compared to control cells (Figure 4A-C). These observations illustrate a link between AATBC, mitochondrial network connectivity, and increased thermogenic function. As mitochondrial fusion is mediated in part by OPA1 and MFN2, we evaluated their protein levels. We found that compared to control cells the levels of both proteins were higher in hMADS cells upon knockdown of AATBC (Figure 4E-G), indicating a shift in mitochondrial dynamics towards fusion. We also measured protein levels of the different OXPHOS complexes, which remained unchanged except for complex $V$ (ATP synthase), which was lower in hMADS cells with silencing of AATBC (Figure $4 \mathrm{H}, \mathrm{I}$ ). Complementary to this data set, overexpression of AATBC was associated with less mitochondrial connectivity (Figure 4J-M) and lower OPA1 but 
unchanged MFN2 and OXPHOS levels compared to control-infected cells (Figure 4N-R). In summary, these data demonstrate that modulation of AATBC expression regulates mitochondrial dynamics.

\section{Expressing human AATBC in mice impacts plasma leptin and triglyceride levels.}

AATBC is specific to humans, which complicates preclinical in vivo investigations in mouse models of metabolic disorders. Regardless, in a first attempt to overcome this obstacle we expressed AATBC in adipose tissue by surgically guided injection of adenovirus (AV) directly into interscapular BAT and inguinal subcutaneous (SCAT) adipose tissue of lean wild-type mice and subsequently performed several metabolic analyses (Figure $5 A$ ). In mice that received AATBC$A V$ we found marked, localized expression of AATBC IncRNA levels in both BAT and SCAT while in GWAT and liver only minor expression was detectable compared to control injected mice (Figure 5B). While body weight, glucose, and insulin tolerance as well as plasma insulin and adiponectin levels remained unchanged, we observed lower leptin levels (Figure 5C-I). In addition, in mice expressing AATBC we found higher plasma triglycerides while non-esterified fatty acids levels were unaltered (Figure $5 \mathrm{~J}, \mathrm{~K}$ ). However, the gene expression levels of Ucp 1 and adiponectin (Adipoq) were not affected (Figure 5L,M), indicating that the treatment has little to no deleterious effects. In line with the lower plasma leptin levels, also gene expression of leptin (Lep) was reduced in scWAT (Figure $5 \mathrm{~N}$ ). In conclusion, our proof-of-concept experiment shows $A A T B C$ expression in mouse adipose tissue influences systemic metabolism in vivo.

\section{AATBC is a novel obesity linked human IncRNA.}

To further corroborate the importance of adipocyte AATBC in systemic metabolism, we investigated the correlation of AATBC with different metabolic parameters in human obesity. Using cross sectional studies with subjects presenting a broad range of body mass index (BMI), we analyzed gene expression of AATBC by $\mathrm{qPCR}$. In two independent cohorts we found that AATBC was expressed at higher levels in visceral (vis)WAT compared to scWAT (Figure 6A, B). Furthermore, the expression of AATBC was lower in visWAT of obese female subjects compared to lean controls (Figure 6B,D,E), an effect not found in scWAT (Figure 6C,F). In another separate cohort that included mainly obese subjects we were able to perform more in-depth analyses as in addition to anthropomorphic and plasma parameters, RNAseq data from adipose tissue samples was available. We found that AATBC was positively regulated with age and negatively regulated with body weight, body fat and the hip-waist ratio in visWAT of both females and males. Similar to our observation in mice, plasma levels of leptin were inversely correlated with AATBC, whereas 
adiponectin levels showed no correlation (Figure 6 G-J). In line with our previous observations, we found that AATBC was positively correlated with thermogenic markers such as UCP1 and PPARG1A (Figure 7, A, B, D, E) and negatively correlated with the expression of genes coding for ADIPOQ, LEP, FABP4 and PPARG. (Figure 7, C, F, G-L). These gene expression correlations were also found in scWAT but in the absence of correlations with the metabolic parameters (Supplementary figures 6 and 7). In summary, adipose AATBC is inversely linked to excess adipose tissue and leptin levels as well as correlates with thermogenic markers in humans.

\section{Discussion:}

Adipocyte plasticity is a key component for the adaptation of metabolism to environmental cues. Thus, the functional features of the adipocyte are matched to the metabolic needs of the organism. This is especially prominent during the adaptation to cold, during which adipocytes adopt a more thermogenic phenotype, or, conversely, when temperature increases or other conditions of positive energy balance such as high-fat diet-induced obesity. If the energy balance is positive, whitening prevails over browning and the adipocytes undergo significant morphological and functional remodeling. Here, we show that the obesity-linked IncRNA AATBC is a regulator of adipocyte plasticity that enhances mitochondrial function in human adipocytes.

Mechanistic metabolic studies in humans are challenging and are further complicated if the gene of interest is absent in mice. To overcome these obstacles, we took advantage of hMADS cells which are a representative model of human adipocytes with the potential to switch between white and thermogenic phenotypes and confirmed our findings in primary cells isolated from human WAT and BAT. We demonstrate that in cultured human adipocytes, AATBC stimulates mitochondrial fission, oxygen consumption, and induces the expression of several markers of browning. Its nuclear localization suggests that AATBC exerts its effects by modulation of transcriptional events, but the outcomes of both loss and gain of AATBC function experiments on the global transcriptome were marginal and not linked to changes in genes that would explain the effects of AATBC on mitochondrial function [70]. Therefore, a non-transcriptional function for AATBC seems more likely. Indeed, IncRNAs can shuttle out of the nucleus and, for example, LNC00473 has been shown to physically present at the lipid droplet-mitochondrial interface [47]. However, we did not observe such a behavior for AATBC, as it remained strictly nuclear.

Likewise, lipolysis is a key feature of adipocyte function and we found that AATBC influences lipolysis both in vitro and in vivo. These functional effects were also mirrored in our adipocyte 
transcriptomic analysis of inhibition and overexpression of AATBC, potentially indicating that AATBC might have a dual role the regulation of adipocyte function. Lipolysis and mitochondrial function are two important pillars of adipocyte plasticity that are rapidly regulated but also change during chronic adaptations to cold or high-fat diet feeding $[20,71]$. To investigate if AATBC is sufficient to alter adipocyte function in vivo, we expressed AATBC by a viral strategy in mice where $A A T B C$ is naturally absent. We found that AATBC expression altered plasma triglyceride and leptin levels, two important systemic parameters of adipocyte function. One could argue that expressing AATBC in mouse adipocyte might be artificial, as AATBC is a human IncRNA and could be non-functional in a mouse cell. However, it has been shown that expressing human IncRNAs in mice is useful for investigating IncRNA function [72] and we did not observe any adverse side effects in mice expressing AATBC. While more work is certainly needed to study the role of AATBC in non-shivering thermogenesis and/or obesity, ideally using a stable transgenic mouse model, our work here indicates that adipocyte AATBC plays role in systemic metabolism.

In humans, adipose AATBC expression is correlated with a thermogenic phenotype. This was also reflected in human primary adipocytes and hMADS cells. Regardless, AATBC is more expressed in visWAT compared to scWAT both in lean and obese patients, which implies that AATBC plays an important role in white adipocytes, too. In line with this notion, AATBC expression was correlated inversely with parameters of obesity and plasma leptin levels. Mitochondrial dynamics are equally important for proper white adipocyte function, as patients with mutations in MFN2 display a fragmented mitochondrial network, excess adiposity, and paradoxical suppression of leptin expression [73]. In general, proper mitochondrial dynamics are critical for maintaining a healthy pool of mitochondria and efficient mitophagy [74]. To this end, it will be intriguing to study the role of AATBC in obesity-induced mitochondrial dysfunction, in adipocyte and potentially also other cell types as AATBC expression is not restricted to adipocytes. In conclusion, our study identifies AATBC as a novel IncRNA linked to adipocyte function and obesity with the potential to manipulate mitochondrial function and thermogenic plasticity in humans. 


\section{Acknowledgements:}

We thank Ez-Zoubir Amri (Institut de Biologie Valrose, Université Nice Sophia Antipolis, France) for sharing hMADS cells. We thank Daniela Hass for outstanding technical support as well as Florian Pauler for valuable advice on the lincRNA RNAseq analysis. We thank the members of Bartelt Lab for enjoyable atmosphere and stimulating discussions. The figures were created using BioRender. MG was supported by an Alexander von Humboldt Foundation postdoctoral fellowship. P.F.P. was supported by the Deutsche Forschungsgemeinschaft (FI 1700/7-1, Heisenberg program). D.T. was supported by a grant from the German Research Association (TE912/2-2). S.H. was supported by the Helmholtz Future topic 'Aging and Metabolic Programming, AMPro', by the Deutsche Forschungsgemeinschaft Trans-Regio (TRR205) and the Deutsches Zentrum für Herz-Kreislauf-Forschung Standortprojekt Cardiometabolism. A.B. was supported by the Deutsche Forschungsgemeinschaft Sonderforschungsbereich 1123 (B10), the Deutsches Zentrum für Herz-Kreislauf-Forschung Junior Research Group Grant, and the European Research Council (ERC) Starting Grant PROTEOFIT. We apologize to colleagues whose work we could not cite due to space limitations.

\section{Conflicts of interest:}

The authors declare no conflicts of interest and that there are no relationships or activities that might bias, or be perceived to bias, their work.

\section{Author contribution statement:}

M.G., S.Ko., M.S., S.H. and A.B. conceptualized the study. M.G., S.Ko., and A.B. wrote the manuscript. M.G., S.Ko, and A.B. interpreted the work. M.G., S.Ko., Y.K., O.LT., M.G.L., A.H, M.K., J.H., S.Kh., D.T., P.F.P., W.S., AG, C.W., M.W., K.A.W., M.B., S.N., A.Z., C.G.C., M.S., S.H. and A.B. contributed to the acquisition and analysis of the data. All the authors revised the manuscript and approved the final version of the manuscript. 


\section{References:}

1. U Din M, Saari T, Raiko J, et al (2018) Postprandial Oxidative Metabolism of Human Brown Fat Indicates Thermogenesis. Cell Metabolism 28(2):207-216.e3. https://doi.org/10.1016/j.cmet.2018.05.020

2. van Marken Lichtenbelt WD, Vanhommerig JW, Smulders NM, et al (2009) ColdActivated Brown Adipose Tissue in Healthy Men. N Engl J Med 360(15):1500-1508. https://doi.org/10.1056/NEJMoa0808718

3. Leitner BP, Huang S, Brychta RJ, et al (2017) Mapping of human brown adipose tissue in lean and obese young men. Proc Natl Acad Sci USA 114(32):8649-8654.

https://doi.org/10.1073/pnas.1705287114

4. Chondronikola M, Volpi E, Børsheim E, et al (2014) Brown Adipose Tissue Improves Whole-Body Glucose Homeostasis and Insulin Sensitivity in Humans. Diabetes 63(12):4089-4099. https://doi.org/10.2337/db14-0746

5. Bartelt A, Heeren J (2014) Adipose tissue browning and metabolic health. Nat Rev Endocrinol 10(1):24-36. https://doi.org/10.1038/nrendo.2013.204

6. Ukropec J, Anunciado RP, Ravussin Y, Hulver MW, Kozak LP (2006) UCP1-independent thermogenesis in white adipose tissue of cold-acclimated Ucp1-/- mice. J Biol Chem 281(42):31894-31908. https://doi.org/10.1074/jbc.M606114200

7. de Meis L (2001) Uncoupled ATPase Activity and Heat Production by the Sarcoplasmic Reticulum Ca2+-ATPase. Journal of Biological Chemistry 276(27):25078-25087. https://doi.org/10.1074/jbc.M103318200

8. Tajima K, Ikeda K, Tanabe Y, et al (2020) Wireless optogenetics protects against obesity via stimulation of non-canonical fat thermogenesis. Nat Commun 11(1):1730. https://doi.org/10.1038/s41467-020-15589-y

9. Anunciado-Koza R, Ukropec J, Koza RA, Kozak LP (2008) Inactivation of UCP1 and the glycerol phosphate cycle synergistically increases energy expenditure to resist diet-induced obesity. J Biol Chem 283(41):27688-27697. https://doi.org/10.1074/jbc.M804268200

10. Keipert S, Kutschke M, Ost M, et al (2017) Long-Term Cold Adaptation Does Not Require FGF21 or UCP1. Cell Metabolism 26(2):437-446.e5. https://doi.org/10.1016/j.cmet.2017.07.016

11. Virtanen KA, Lidell ME, Orava J, et al (2009) Functional Brown Adipose Tissue in Healthy Adults. N Engl J Med 360(15):1518-1525. https://doi.org/10.1056/NEJMoa0808949

12. Shimizu I, Walsh K (2015) The Whitening of Brown Fat and Its Implications for Weight Management in Obesity. Curr Obes Rep 4(2):224-229. https://doi.org/10.1007/s13679-0150157-8 
13. Cannon B, Nedergaard J (2011) Nonshivering thermogenesis and its adequate measurement in metabolic studies. J Exp Biol 214(Pt 2):242-253. https://doi.org/10.1242/jeb.050989

14. de Jong JMA, Sun W, Pires ND, et al (2019) Human brown adipose tissue is phenocopied by classical brown adipose tissue in physiologically humanized mice. Nat Metab 1(8):830 843. https://doi.org/10.1038/s42255-019-0101-4

15. Bartelt A, John C, Schaltenberg N, et al (2017) Thermogenic adipocytes promote HDL turnover and reverse cholesterol transport. Nat Commun 8:15010. https://doi.org/10.1038/ncomms15010

16. Bartelt A, Bruns OT, Reimer R, et al (2011) Brown adipose tissue activity controls triglyceride clearance. Nat Med 17(2):200-205. https://doi.org/10.1038/nm.2297

17. Berbée JFP, Boon MR, Khedoe PPSJ, et al (2015) Brown fat activation reduces hypercholesterolaemia and protects from atherosclerosis development. Nat Commun 6:6356. https://doi.org/10.1038/ncomms 7356

18. Becher T, Palanisamy S, Kramer DJ, et al (2021) Brown adipose tissue is associated with cardiometabolic health. Nat Med 27(1):58-65. https://doi.org/10.1038/s41591-020-1126-7

19. Giacomello M, Pyakurel A, Glytsou C, Scorrano L (2020) The cell biology of mitochondrial membrane dynamics. Nat Rev Mol Cell Biol 21(4):204-224. https://doi.org/10.1038/s41580-020-0210-7

20. Dai W, Jiang L (2019) Dysregulated Mitochondrial Dynamics and Metabolism in Obesity, Diabetes, and Cancer. Front Endocrinol 10:570. https://doi.org/10.3389/fendo.2019.00570

21. Rogne M, Chu D-T, Küntziger TM, Mylonakou M-N, Collas P, Tasken K (2018) OPA1anchored PKA phosphorylates perilipin 1 on S522 and S497 in adipocytes differentiated from human adipose stem cells. MBoC 29(12):1487-1501. https://doi.org/10.1091/mbc.E17-09-0538

22. Quirós PM, Ramsay AJ, Sala D, et al (2012) Loss of mitochondrial protease OMA1 alters processing of the GTPase OPA1 and causes obesity and defective thermogenesis in mice: Metabolic control by mitochondrial protease OMA1. The EMBO Journal 31(9):2117-2133. https://doi.org/10.1038/emboj.2012.70

23. Pisani DF, Barquissau V, Chambard J-C, et al (2018) Mitochondrial fission is associated with UCP1 activity in human brite/beige adipocytes. Mol Metab 7:35-44. https://doi.org/10.1016/j.molmet.2017.11.007

24. Puigserver P, Wu Z, Park CW, Graves R, Wright M, Spiegelman BM (1998) A coldinducible coactivator of nuclear receptors linked to adaptive thermogenesis. Cell 92(6):829-839. https://doi.org/10.1016/s0092-8674(00)81410-5 
25. Bartelt A, Widenmaier SB, Schlein C, et al (2018) Brown adipose tissue thermogenic adaptation requires Nrf1-mediated proteasomal activity. Nat Med 24(3):292-303. https://doi.org/10.1038/nm.4481

26. Wikstrom JD, Mahdaviani K, Liesa M, et al (2014) Hormone-induced mitochondrial fission is utilized by brown adipocytes as an amplification pathway for energy expenditure. EMBO J 33(5):418-436. https://doi.org/10.1002/embj.201385014

27. Boutant M, Kulkarni SS, Joffraud M, et al (2017) Mfn2 is critical for brown adipose tissue thermogenic function. EMBO J 36(11):1543-1558.

https://doi.org/10.15252/embj.201694914

28. Mahdaviani K, Benador IY, Su S, et al (2017) Mfn2 deletion in brown adipose tissue protects from insulin resistance and impairs thermogenesis. EMBO Rep 18(7):1123-1138. https://doi.org/10.15252/embr.201643827

29. Pereira RO, Marti A, Olvera AC, et al (2021) OPA1 deletion in brown adipose tissue improves thermoregulation and systemic metabolism via FGF21. Elife 10:e66519. https://doi.org/10.7554/eLife.66519

30. Sun L, Lin JD (2019) Function and Mechanism of Long Noncoding RNAs in Adipocyte Biology. Diabetes 68(5):887-896. https://doi.org/10.2337/dbi18-0009

31. Schmidt E, Dhaouadi I, Gaziano I, et al (2018) LincRNA H19 protects from dietary obesity by constraining expression of monoallelic genes in brown fat. Nat Commun 9(1):3622. https://doi.org/10.1038/s41467-018-05933-8

32. Dallner OS, Marinis JM, Lu Y-H, et al (2019) Dysregulation of a long noncoding RNA reduces leptin leading to a leptin-responsive form of obesity. Nat Med 25(3):507-516. https://doi.org/10.1038/s41591-019-0370-1

33. Lo KA, Huang S, Walet ACE, et al (2018) Adipocyte Long-Noncoding RNA Transcriptome Analysis of Obese Mice Identified Lnc-Leptin, Which Regulates Leptin. Diabetes 67(6):1045-1056. https://doi.org/10.2337/db17-0526

34. Zhang B, Xu S, Liu J, Xie Y, Xiaobo S (2021) Long Noncoding RNAs: Novel Important Players in Adipocyte Lipid Metabolism and Derivative Diseases. Front Physiol 12:691824. https://doi.org/10.3389/fphys.2021.691824

35. Eisenberg E, Levanon EY (2013) Human housekeeping genes, revisited. Trends Genet 29(10):569-574. https://doi.org/10.1016/j.tig.2013.05.010

36. Kazimierczyk M, Kasprowicz MK, Kasprzyk ME, Wrzesinski J (2020) Human Long Noncoding RNA Interactome: Detection, Characterization and Function. Int J Mol Sci 21(3):E1027. https://doi.org/10.3390/ijms21031027 
37. MacDonald WA, Mann MRW (2020) Long noncoding RNA functionality in imprinted domain regulation. PLoS Genet 16(8):e1008930. https://doi.org/10.1371/journal.pgen.1008930

38. Statello L, Guo C-J, Chen L-L, Huarte M (2021) Gene regulation by long non-coding RNAs and its biological functions. Nat Rev Mol Cell Biol 22(2):96-118. https://doi.org/10.1038/s41580-020-00315-9

39. Giroud M, Scheideler M (2017) Long Non-Coding RNAs in Metabolic Organs and Energy Homeostasis. Int J Mol Sci 18(12):E2578. https://doi.org/10.3390/ijms18122578

40. Kornfeld J-W, Brüning JC (2014) Regulation of metabolism by long, non-coding RNAs. Front Genet 5:57. https://doi.org/10.3389/fgene.2014.00057

41. Ji E, Kim C, Kim W, Lee EK (2020) Role of long non-coding RNAs in metabolic control. Biochim Biophys Acta Gene Regul Mech 1863(4):194348. https://doi.org/10.1016/j.bbagrm.2018.12.006

42. Zhao X-Y, Li S, Wang G-X, Yu Q, Lin JD (2014) A long noncoding RNA transcriptional regulatory circuit drives thermogenic adipocyte differentiation. Mol Cell 55(3):372-382. https://doi.org/10.1016/j.molcel.2014.06.004

43. Alvarez-Dominguez JR, Bai Z, Xu D, et al (2015) De Novo Reconstruction of Adipose Tissue Transcriptomes Reveals Long Non-coding RNA Regulators of Brown Adipocyte Development. Cell Metab 21(5):764-776. https://doi.org/10.1016/j.cmet.2015.04.003

44. Bai Z, Chai X, Yoon MJ, et al (2017) Dynamic transcriptome changes during adipose tissue energy expenditure reveal critical roles for long noncoding RNA regulators. PLoS Biol 15(8):e2002176. https://doi.org/10.1371/journal.pbio.2002176

45. Bast-Habersbrunner A, Kiefer C, Weber P, et al (2021) LncRNA Ctcflos orchestrates transcription and alternative splicing in thermogenic adipogenesis. EMBO Rep 22(7). https://doi.org/10.15252/embr.202051289

46. Ding C, Lim YC, Chia SY, et al (2018) De novo reconstruction of human adipose transcriptome reveals conserved lncRNAs as regulators of brown adipogenesis. Nat Commun 9(1):1329. https://doi.org/10.1038/s41467-018-03754-3

47. Tran K-V, Brown EL, DeSouza T, et al (2020) Human thermogenic adipocyte regulation by the long noncoding RNA LINC00473. Nat Metab 2(5):397-412. https://doi.org/10.1038/s42255-020-0205-x

48. Tang T, Yang L, Cao Y, et al (2020) LncRNA AATBC regulates Pinin to promote metastasis in nasopharyngeal carcinoma. Mol Oncol 14(9):2251-2270. https://doi.org/10.1002/1878-0261.12703 
49. Wang M, Dai M, Wang D, et al (2021) The long noncoding RNA AATBC promotes breast cancer migration and invasion by interacting with YBX1 and activating the YAP1/Hippo signaling pathway. Cancer Lett 512:60-72. https://doi.org/10.1016/j.canlet.2021.04.025

50. Zhao F, Lin T, He W, et al (2015) Knockdown of a novel lincRNA AATBC suppresses proliferation and induces apoptosis in bladder cancer. Oncotarget 6(2):1064-1078. https://doi.org/10.18632/oncotarget.2833

51. Yan K, Wang Y, Shao Y, Xiao T (2021) Gene Instability-Related lncRNA Prognostic Model of Melanoma Patients via Machine Learning Strategy. J Oncol 2021:5582920. https://doi.org/10.1155/2021/5582920

52. Higareda-Almaraz JC, Karbiener M, Giroud M, et al (2018) Norepinephrine triggers an immediate-early regulatory network response in primary human white adipocytes. BMC Genomics 19(1):794. https://doi.org/10.1186/s12864-018-5173-0

53. Giroud M, Tsokanos F-F, Caratti G, et al (2021) HAND2 is a novel obesity-linked adipogenic transcription factor regulated by glucocorticoid signalling. Diabetologia 64(8):1850-1865. https://doi.org/10.1007/s00125-021-05470-y

54. Jespersen NZ, Larsen TJ, Peijs L, et al (2013) A classical brown adipose tissue mRNA signature partly overlaps with brite in the supraclavicular region of adult humans. Cell Metab 17(5):798-805. https://doi.org/10.1016/j.cmet.2013.04.011

55. Schmieder R, Edwards R (2011) Quality control and preprocessing of metagenomic datasets. Bioinformatics 27(6):863-864. https://doi.org/10.1093/bioinformatics/btr026

56. Giroud M, Pisani DF, Karbiener M, et al (2016) miR-125b affects mitochondrial biogenesis and impairs brite adipocyte formation and function. Mol Metab 5(8):615-625.

https://doi.org/10.1016/j.molmet.2016.06.005

57. Kirshner H, Aguet F, Sage D, Unser M (2013) 3-D PSF fitting for fluorescence microscopy: implementation and localization application. J Microsc 249(1):13-25. https://doi.org/10.1111/j.1365-2818.2012.03675.x

58. Sage D, Donati L, Soulez F, et al (2017) DeconvolutionLab2: An open-source software for deconvolution microscopy. Methods 115:28-41. https://doi.org/10.1016/j.ymeth.2016.12.015

59. Seitz S, Kwon Y, Hartleben G, et al (2019) Hepatic Rab24 controls blood glucose homeostasis via improving mitochondrial plasticity. Nat Metab 1(10):1009-1026. https://doi.org/10.1038/s42255-019-0124-x

60. Picelli S, Faridani OR, Björklund ÅK, Winberg G, Sagasser S, Sandberg R (2014) Fulllength RNA-seq from single cells using Smart-seq2. Nat Protoc 9(1):171-181. https://doi.org/10.1038/nprot.2014.006 
61. Song Y, Milon B, Ott S, et al (2018) A comparative analysis of library prep approaches for sequencing low input translatome samples. BMC Genomics 19(1):696. https://doi.org/10.1186/s12864-018-5066-2

62. Dobin A, Gingeras TR (2015) Mapping RNA-seq Reads with STAR. Curr Protoc Bioinformatics 51:11.14.1-11.14.19. https://doi.org/10.1002/0471250953.bi1114s51

63. Liao Y, Smyth GK, Shi W (2014) featureCounts: an efficient general purpose program for assigning sequence reads to genomic features. Bioinformatics 30(7):923-930. https://doi.org/10.1093/bioinformatics/btt656

64. Love MI, Huber W, Anders S (2014) Moderated estimation of fold change and dispersion for RNA-seq data with DESeq2. Genome Biol 15(12):550. https://doi.org/10.1186/s13059014-0550-8

65. Chen S, Zhou Y, Chen Y, Gu J (2018) fastp: an ultra-fast all-in-one FASTQ preprocessor. Bioinformatics 34(17):i884-i890. https://doi.org/10.1093/bioinformatics/bty560

66. Frankish A, Diekhans M, Ferreira A-M, et al (2019) GENCODE reference annotation for the human and mouse genomes. Nucleic Acids Research 47(D1):D766-D773. https://doi.org/10.1093/nar/gky955

67. Dobin A, Davis CA, Schlesinger F, et al (2013) STAR: ultrafast universal RNA-seq aligner. Bioinformatics 29(1):15-21. https://doi.org/10.1093/bioinformatics/bts635

68. Petrovic N, Walden TB, Shabalina IG, Timmons JA, Cannon B, Nedergaard J (2010) Chronic peroxisome proliferator-activated receptor gamma (PPARgamma) activation of epididymally derived white adipocyte cultures reveals a population of thermogenically competent, UCP1-containing adipocytes molecularly distinct from classic brown adipocytes. J Biol Chem 285(10):7153-7164. https://doi.org/10.1074/jbc.M109.053942

69. Thaher O, Wolf C, Dey PN, et al (2018) The thiol switch C684 in Mitofusin-2 mediates redox-induced alterations of mitochondrial shape and respiration. Neurochemistry International 117:167-173. https://doi.org/10.1016/j.neuint.2017.05.009

70. Chen L-L (2016) Linking Long Noncoding RNA Localization and Function. Trends in Biochemical Sciences 41(9):761-772. https://doi.org/10.1016/j.tibs.2016.07.003

71. Weir G, Ramage LE, Akyol M, et al (2018) Substantial Metabolic Activity of Human Brown Adipose Tissue during Warm Conditions and Cold-Induced Lipolysis of Local Triglycerides. Cell Metabolism 27(6):1348-1355.e4. https://doi.org/10.1016/j.cmet.2018.04.020

72. Ruan X, Li P, Chen Y, et al (2020) In vivo functional analysis of non-conserved human lncRNAs associated with cardiometabolic traits. Nat Commun 11(1):45. https://doi.org/10.1038/s41467-019-13688-z 
73. Rocha N, Bulger DA, Frontini A, et al (2017) Human biallelic MFN2 mutations induce mitochondrial dysfunction, upper body adipose hyperplasia, and suppression of leptin expression. Elife 6:e23813. https://doi.org/10.7554/eLife.23813

74. Liesa M, Shirihai OS (2013) Mitochondrial dynamics in the regulation of nutrient utilization and energy expenditure. Cell Metab 17(4):491-506.

https://doi.org/10.1016/j.cmet.2013.03.002 


\section{Figure Legends:}

Figure 1: Identification of the IncRNA AATBC linked to thermogenesis in humans

(A) Experimental outline (B) Venn diagram of regulated IncRNA (C-E) UCP1 expression in (C) human brown and white adipose tissue, (D) human primary adipocytes treated with $1 \mu \mathrm{M}$ norepinephrine for 6 hours and (E) hMADS cells treated at day 15 of differentiation with $1 \mu \mathrm{M}$ forskolin for $6 \mathrm{~h}$ (C: $n=5$ patients, D: $n=5$ replicates, E: $n=1$ replicate) (F) Heatmap of the differential expression of IncRNAs ( $n=1-3$ replicates) (G-L) Expression of AATBC and LINC00473 (G,H: $n=5$ subjects; I,J: $n=5$ replicates; K,L: $n=1$ replicate).

Figure 2: AATBC is associated with adipocyte browning and found in the nucleus (A, B) AATBC expression in (A) white or (B) brown human stromal-vascular fraction (hSVF) cells treated with $1 \mu \mathrm{M}$ norepinephrine for $6 \mathrm{~h}$ ( $n=5$ replicates) (C) AATBC expression in hSVF differentiated to a white and thermogenic phenotype ( $n=7$ replicates) (D) AATBC expression in the SVF, adipocyte fraction (AF) and total fraction (TF) of human white adipose tissue $(n=9$ replicates) (E,F) AATBC expression in (E) white or $(F)$ thermogenic human mesenchymal adipose stem (hMADS) cells treated with $1 \mu \mathrm{M}$ norepinephrine for $6 \mathrm{~h}$ ( $n=9$ replicates) (G) AATBC expression in hMADS cells differentiated to a white and thermogenic phenotype ( $n=9$ replicates) (H) AATBC expression in undifferentiated and differentiated hMADS cells ( $n=12$ replicates) (IK) Expression of $A A T B C, 12 S$ and U1 mRNA in the nucleus and cytosol of thermogenic hMADS cells ( $n=8$ replicates) (L,M) In situ hybridization (RNAScope $\AA$ ) of $A A T B C$ in thermogenic hMADS cells at $(L)$ untreated conditions and (M) in thermogenic hMADS treated with adenovirus (AV)mediated overexpression (OE) of AATBC (or control $A V$ ) (representative images from 3 independent experiments) (M). Scale bars: $20 \mu \mathrm{m}$ and $5 \mu \mathrm{m}$ for the magnified images. Statistical significance ${ }^{*} p<0.05$ by 2 -tailed, unpaired Student's T-test $(\mathbf{A}, \mathbf{C}-\mathbf{K})$ or 2-way ANOVA followed by Tukey's test (B).

\section{Figure 3: AATBC is a regulator of mitochondrial function}

(A) Experimental outline (B, C) Expression of $A A T B C$ and UCP1 during anti-sense oligonucleotide (ASO)-mediated knockdown of AATBC ( $n=9-15$ replicates) (D-H) Oxygen consumption rate (OCR) during ASO-mediated knockdown of AATBC visualized as $(\mathrm{H})$ trace or cumulative at $(D)$ baseline measurement, $(E)$ isoproterenol-induced OCR, $(F)$ oligomycin (Oligo) induced proton leak or (G) carbonyl cyanide-4-(trifluoromethoxy)phenylhydrazone (FCCP)induced maximal respiration (D-G: $n=9$ replicates, $\mathrm{H}$ : representative trace) $(\mathbf{I}, \mathbf{J})$ Expression of 
$A A T B C$ and UCP1 during adenovirus (AV)-mediated overexpression of $A A T B C$ ( $n=8$ replicates) (K-O) OCR during ASO-mediated knockdown of $A A T B C$ visualized as $(\mathrm{O})$ trace or cumulative at (K) baseline measurement, (L) isoproterenol-induced OCR, (M) OCR after oligomycin (Oligo) or (N) FCCP-induced maximal respiration (K-N: $n=27$ replicates, O: representative trace) Statistical significance ${ }^{*} p<0.05$ by 2-way ANOVA followed by Tukey's test (B-G) or 2-tailed unpaired Student's T-test (I-N).

\section{Figure 4: AATBC modulates mitochondrial dynamics by promoting fission}

(A) TOMM20 staining and image analysis of white hMADS cells (representative image of 3 independent experiments) (B-D) Quantification of (B) length of mitochondrial branches, (C) junctions per cell area and (D) branches per cell area ( $n=24$ cells) (E-G) Immunoblot of OPA1, MFN2 and $\beta$-tubulin and the respective quantification normalized to $\beta$-Tubulin levels ( $E$ : representative immunoblot; F,G: $n=15$ replicates) (H,I) Immunoblot of OXPHOS complexes and normalized to $\beta$-Tubulin levels ( $\mathrm{H}$ : representative immunoblot; I: $n=6$ replicates) $(\mathrm{J})$ TOMM20 staining and image analysis of white hMADS cells (representative image of 3 independent experiments) (K-M) Quantification of (K) length of mitochondrial branches, $(\mathrm{L})$ junctions per cell area and (M) branches per cell area ( $n=24$ observations) (N-P) Immunoblot of OPA1, MFN2 and $\beta$-Tubulin and the respective quantification normalized to $\beta$-Tubulin levels ( $N$ : representative immunoblot; O: $n=15$ replicates; P: $n=6$ replicates) (Q,R) Immunoblot of OXPHOS complexes and normalized to $\beta$-tubulin levels (Q: representative immunoblot; R: $n=6$ replicates) Statistical significance * $p<0.05$ by 2-tailed unpaired Student's T-test (B-D,F, G,I,K-M,O,P,R).

\section{Figure 5: Expression of human AATBC in mouse adipose tissue impacts metabolism}

(A) Experimental outline, (B) Expression of $A A T B C$ in adipose tissue depots and liver after injection of AV_GFP or AV_AATBC in brown adipose tissue (BAT) and subcutaneous adipose tissue (scWAT) (C) Body weight (D) Glucose tolerance test (GTT) (E) Insulin tolerance test (ITT) (F-K) plasma levels of metabolic parameters (L-N) Ucp1, Adipoq and Lep gene expression in adipose tissue ( $n=12$ animals per group) Statistical significance ${ }^{*} p<0.05$ by 2-way ANOVA followed by Tukey's test (B,L-N) or 2-tailed unpaired Student's T-test (C,F-K).

\section{Figure 6: $A A T B C$ is linked to obesity and leptin levels in humans}

(A-C) AATBC expression in visceral (visWAT) and subcutaneous (scWAT) white adipose tissue grouped by $(\mathrm{A})$ sex $(\mathrm{B}, \mathrm{C})$ and body mass index (BMI) $(n=318)(\mathrm{D}-\mathrm{F})$ AATBC expression in visWAT and scWAT of $(A)$ male and female subjects $(B, C)$ lean and obese subjects $(n=96$ 
patients) $(\mathbf{G}, \mathbf{H})$ Correlation matrix of $A A T B C$ in visWAT of $(\mathrm{G})$ female and $(\mathrm{H})$ male subjects and metabolic parameters (females: $n=627$, males: $n=293$ ) (I-L) AATBC expression in visWAT of males and females correlated with $(\mathrm{I}, \mathrm{J})$ adiponectin and $(\mathrm{K}, \mathrm{L})$ leptin plasma levels $\left(\mathrm{I}: \log _{\mathrm{e}}(S)=\right.$ 11.02, $p=0.906, \rho_{\text {spearman }}=0.01, \mathrm{Cl}_{95 \%}[-0.22,0.25], n_{\text {pairs }}=72, \mathrm{~J}: \log _{\mathrm{e}}(S)=8.91, p=0.465$, $\rho_{\text {Spearman }}=0.12, \mathrm{Cl}_{95 \%}[-0.22,0.44], n_{\text {pairs }}=37, \mathrm{~K}: \log _{\mathrm{e}}(S)=15.16, p=0.001, \rho_{\text {Spearman }}=-0.21$, $\mathrm{Cl}_{95 \%}[-0.32,-0.09], n_{\text {pairs }}=267, \mathrm{~L}: \log _{\mathrm{e}}(S)=12.80, p=0.011, \rho_{\text {spearman }}=-0.23, \mathrm{Cl}_{95 \%}[-0.40$, $\left.-0.05], n_{\text {pairs }}=121\right)$ Statistical significance ${ }^{*} p<0.05$ by 2-way ANOVA followed by Tukey's test (A-F).

Figure 7: $A A T B C$ correlates with markers of metabolic health in visceral adipose tissue

(A-L) AATBC expression correlated with gene expression of the thermogenic markers PPARGC1A and UCP1 and the obesity-related genes ADIPOQ, FABP4, LEP and PPARG in visceral adipose tissue of $(\mathrm{A}-\mathrm{C}, \mathrm{G}-\mathrm{I})$ females $(n=627)$ and $(\mathrm{D}-\mathrm{F}, \mathrm{J}-\mathrm{L})$ males $(n=293)\left(\mathrm{A}: \log _{\mathrm{e}}(S)\right.$ $=16.47, p=9.09 \mathrm{e}^{-78}, \rho_{\text {spearman }}=0.65, C_{95 \%}[0.61,0.70] ; B: \log _{\mathrm{e}}(S)=16.63, p=1.37 \mathrm{e}^{-60}, \rho_{\text {spearman }}$ $=0.59, \mathrm{Cl}_{95 \%}[0.54,0.64] ; \mathrm{C}: \log _{\mathrm{e}}(S)=17.94, p=3.85 \mathrm{e}^{-43}, \rho_{\text {spearman }}=-0.51, \mathrm{Cl}_{95 \%}[-0.54,-0.45]$; $\mathrm{D}: \log _{\mathrm{e}}(S)=14.30, p=1.04 \mathrm{e}^{-31}, \rho_{\text {Spearman }}=0.61, \mathrm{Cl}_{95 \%}[0.53,0.68] ; \mathrm{E}: \log _{\mathrm{e}}(S)=14.23, p=7.55 \mathrm{e}^{-35}$, $\rho_{\text {Spearman }}=0.64, \mathrm{Cl}_{95 \%}[0.56,0.70] ; \mathrm{F}: \log _{\mathrm{e}}(S)=15.71, p=6.3 \mathrm{e}^{-28}, \rho_{\text {Spearman }}=-0.58, \mathrm{Cl}_{95 \%}[-0.65,-$ 0.50]; $\mathrm{G}: \log _{\mathrm{e}}(S)=17.85, p=4.95 \mathrm{e}^{-22}, \rho_{\mathrm{Spearman}}=-0.37, \mathrm{Cl}_{95 \%}[-0.44,-0.30] ; \mathrm{H}: \log _{\mathrm{e}}(S)=17.89, p$ $=6.46 \mathrm{e}^{-29}, \rho_{\text {Spearman }}=-0.43, \mathrm{Cl}_{95 \%}[-0.49,-0.36] ; \mathrm{I}: \log _{\mathrm{e}}(S)=17.99, p=7.06 \mathrm{e}^{-60}, \rho_{\text {Spearman }}=-0.59$, $\mathrm{Cl}_{95 \%}[-0.64,-0.53] ; \mathrm{J}: \log _{\mathrm{e}}(S)=15.67, p=1.1 \mathrm{e}^{-21}, \rho_{\text {spearman }}=-0.52, \mathrm{Cl}_{95 \%}[-0.60,-0.43] ; \mathrm{K}: \log _{\mathrm{e}}(S)$ $=15.65, p=1.81 \mathrm{e}^{-19}, \rho_{\mathrm{Spearman}}=-0.49, \mathrm{Cl}_{95 \%}[-0.58,-0.40] ; \mathrm{L}: \log _{\mathrm{e}}(S)=15.78, p=2.37 \mathrm{e}^{-43}$, $\left.\rho_{\text {spearman }}=-0.69, \mathrm{Cl}_{95 \%}[-0.75,-0.63]\right)$. 
The obesity-linked human IncRNA AATBC regulates adipocyte plasticity by stimulating mitochondrial dynamics and respiration

Maude Giroud, Stefan Kotschi et al.

Table 1: Antibodies:

\begin{tabular}{|l|l|}
\hline Mfn2 & ab56889 (Abcam) \\
\hline OXPHOS & ab110413 (Abcam) \\
\hline Opa1 & ab42364 (Abcam) \\
\hline ß-tubulin & $2146 S$ (Cell Signaling Technology) \\
\hline TOMM20 & ab78547 (Abcam) \\
\hline Anti-rabbit IgG & $7074 S$ (Cell Signaling Technology) \\
\hline Anti-mouse IgG & 7076S (Cell Signaling Technology) \\
\hline
\end{tabular}

Table 2: Primers:

\begin{tabular}{|l|l|}
\hline h_PLIN1_F & ACCCCCCTGAAAAGATTGCTT \\
\hline h_PLIN1_R & GATGGGAACGCTGATGCTGTT \\
\hline h_TBP_F & ACGCCAGCTTCGGAGAGTTC \\
\hline h_TBP_R & CAAACCGCTTGGGATTATATTCG \\
\hline h_UCP1_F & CTGGACACGGCCAAAGTC \\
\hline h_UCP1_R & GGACACCTTTATACCTAATAACACTGG \\
\hline m_Ucp1_F & AGGCTTCCAGTACCATTAGGT \\
\hline m_Ucp1_R & CTGAGTGAGGCAAAGCTGATTT \\
\hline m_Tbp_F & AGAACAATCCAGACTAGCAGCA \\
\hline m_Tbp_R & GGGAACTTCACATCACAGCTC \\
\hline m_Adipoq_F & GGAGAGAAAGGAGATGCAGGT \\
\hline m_Adipoq_R & CTTTCCTGCCAGGGGTTC \\
\hline h_ADIPOQ_F & GCTGGTCTGAAACTCCTGACA \\
\hline h_ADIPOQ_R & CGGGCAGAGCTAATAGCAGTA \\
\hline h_AATBC_F & ACCGGGCAAATCTGAAACCA 8!!! \\
\hline h_AATBC_R & CGTTGATAACCGGCCTTCCT \\
\hline h_12S_F & CCTGGTCATGAACAAGCAATACC \\
\hline h_12S_R & GTGTCTGGCTAGTGAGGACTG \\
\hline h_U1_F & ATACTTACCTGGCAGGGGAG \\
\hline
\end{tabular}




\begin{tabular}{|l|l|}
\hline h_U1_R & CAGGGGAAAGCGCGAACGCA \\
\hline h_FABP4_F & CCTTTAAAAATACTGAGATTTCCTTCA \\
\hline h_FABP4_R & GGACACCCCCATCTAAGGTT \\
\hline h_NADH_F & GAGCGATGGTGAGAGCTAAGGT \\
\hline h_NADH_R & CCCTAAAACCCGCCACATCT \\
\hline m_Lep_F & CAGGATCAATGACATTTCACACA \\
\hline m_Lep_R & GCTGGTGAGGACCTGTTGAT \\
\hline h_LPL_F & TTCTGGATTCCAATGCTTCGA \\
\hline h_LPL_R & CGAGTCGTCTTTCTCCTGATGAT \\
\hline
\end{tabular}


A

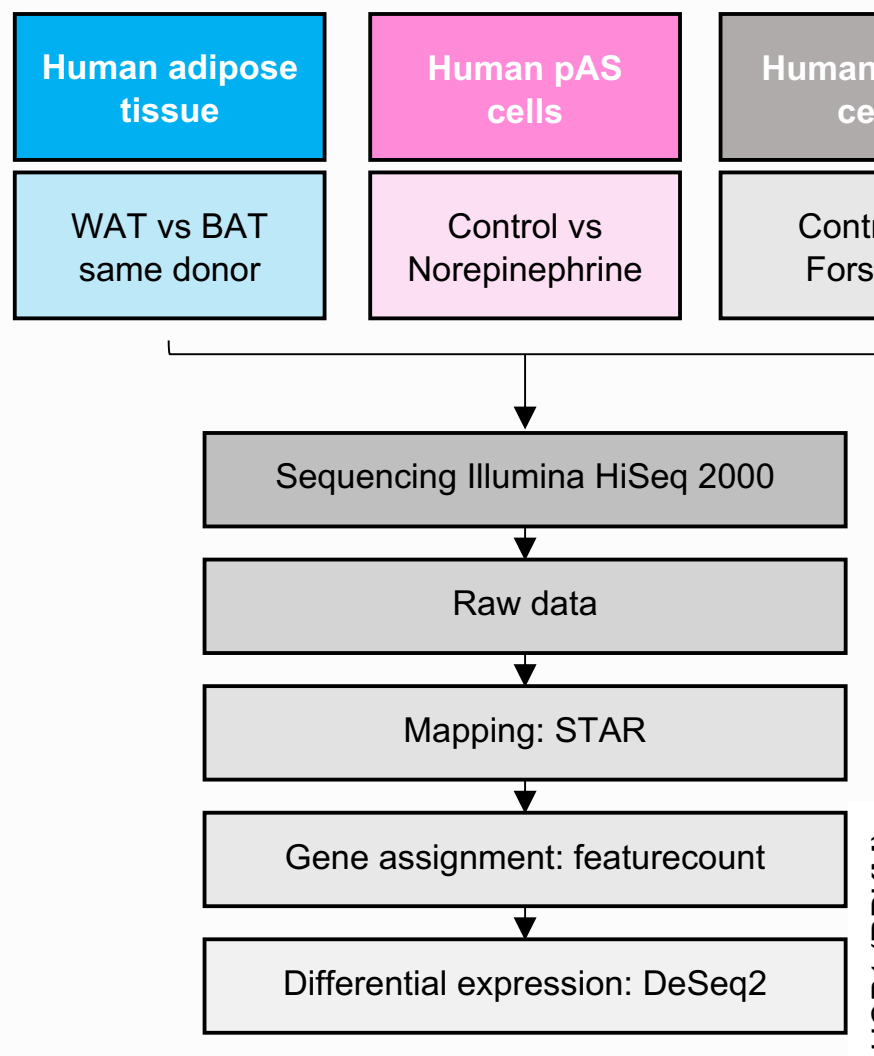

G
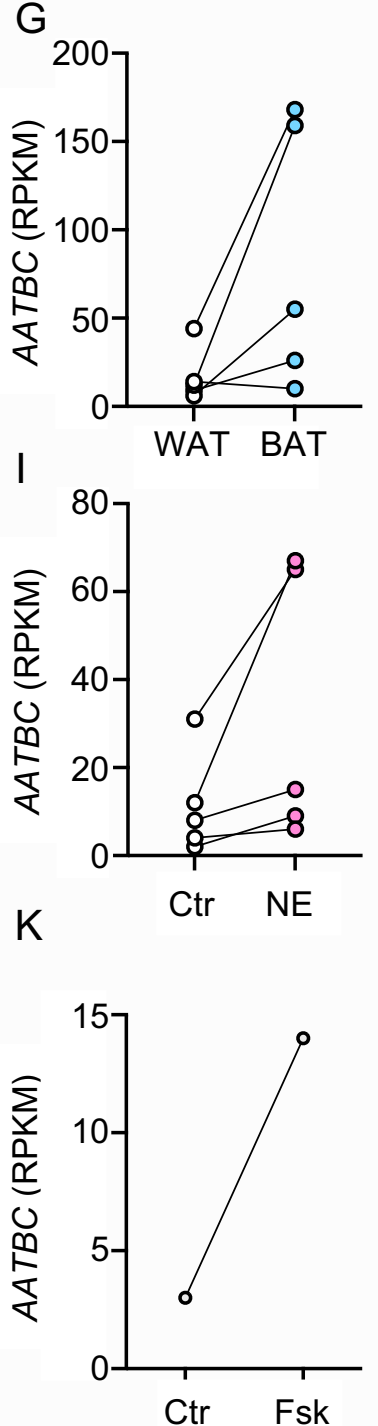

$\mathrm{H}$
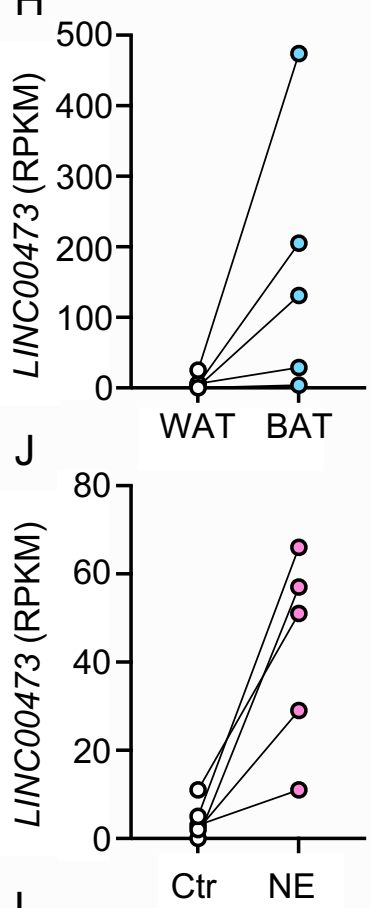

L

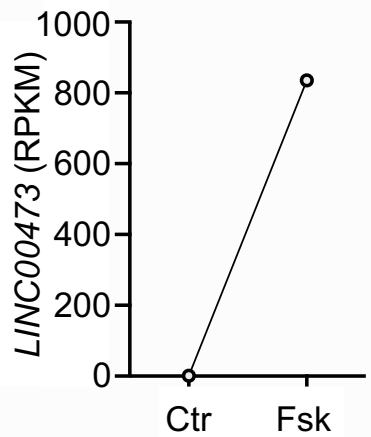

B

Figure 1

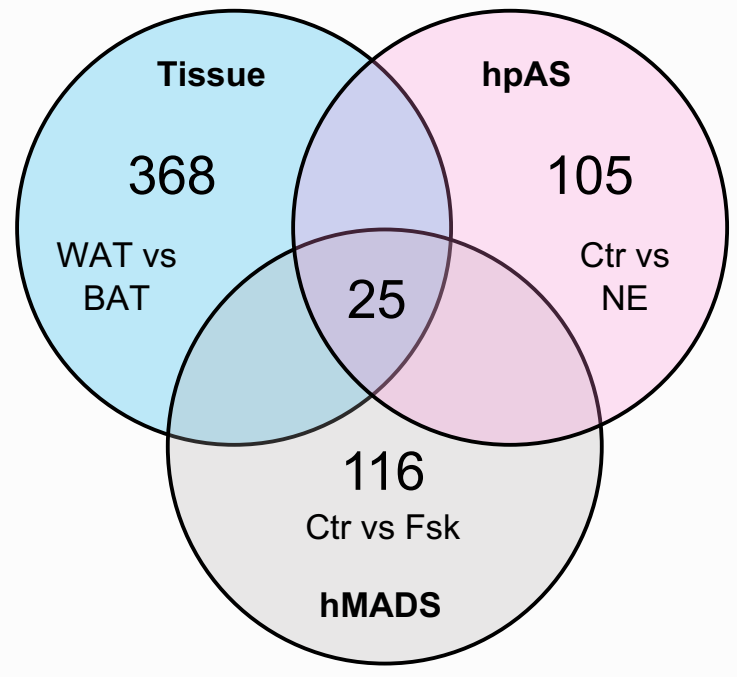

D

E
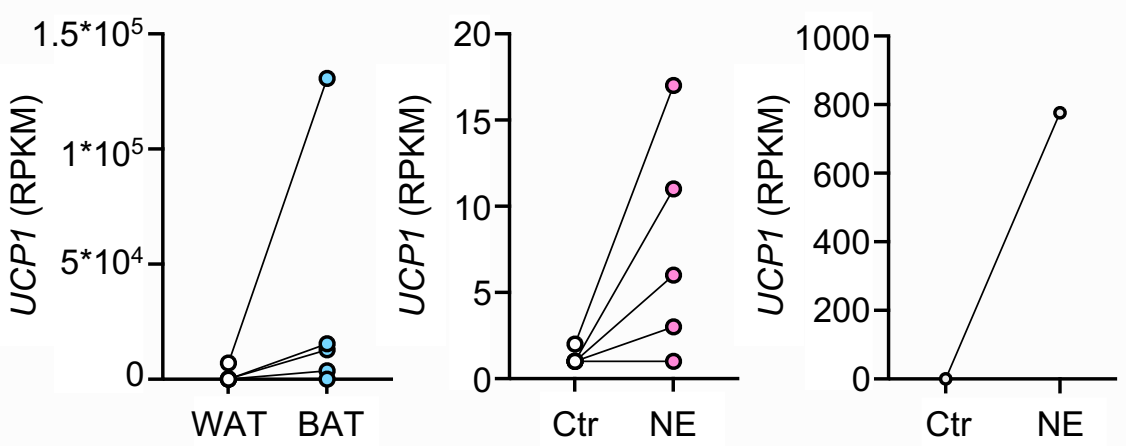

F

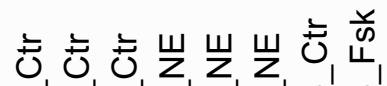

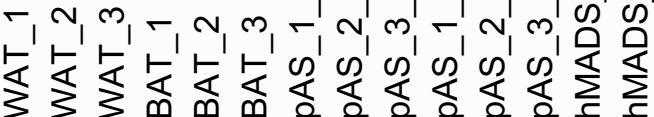

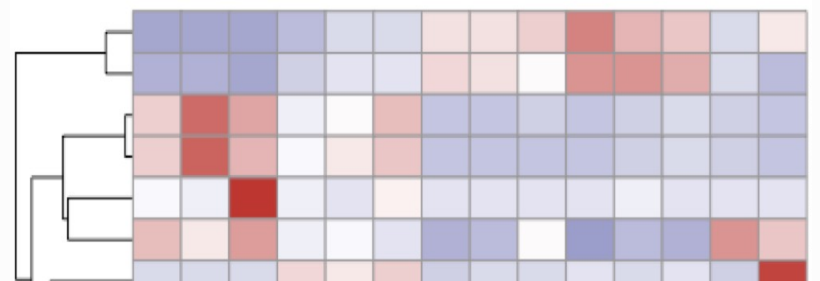

Inc-SPATA5-2:1

Inc-LRFN2-3:1

Inc-ASB3-12:1

XLOC 003415

Inc-ANKRD50-2:1

MIR100HG

LINC00473

AATBC

Inc-MRM2-1:1

LINC01829

VAV3-AS1:16

XLOC_006143

XLOC_001411

LINC01347

Inc-AJAP1-2:1

LINC00847

Inc-PDE4D-1:1

Inc-SLC38A9-1:1

XLOC_005138

XLOC 004873

LINC00847

SNHG16

Inc-USP34-1:1

XLOC_000705

MIRLET7BHG

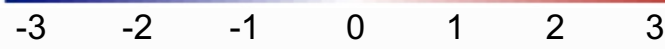


A bioRxiv preprint doi: https://dgiorg/10.1101/2021.08.09.455681; this version posted August 9, 2021. The copyright holder for this preprint Figure 2
(which was not certiked by peer review) is the author/funder. All fights reserved. No reuse allowed withoußpermission.

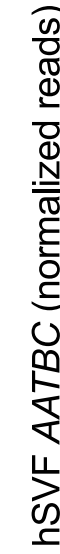

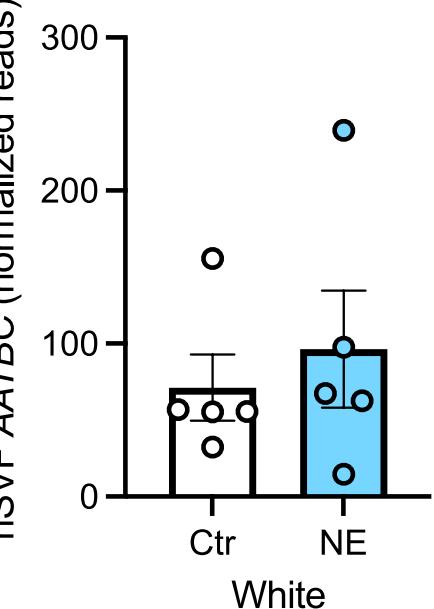

E

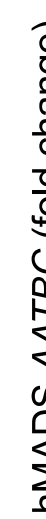
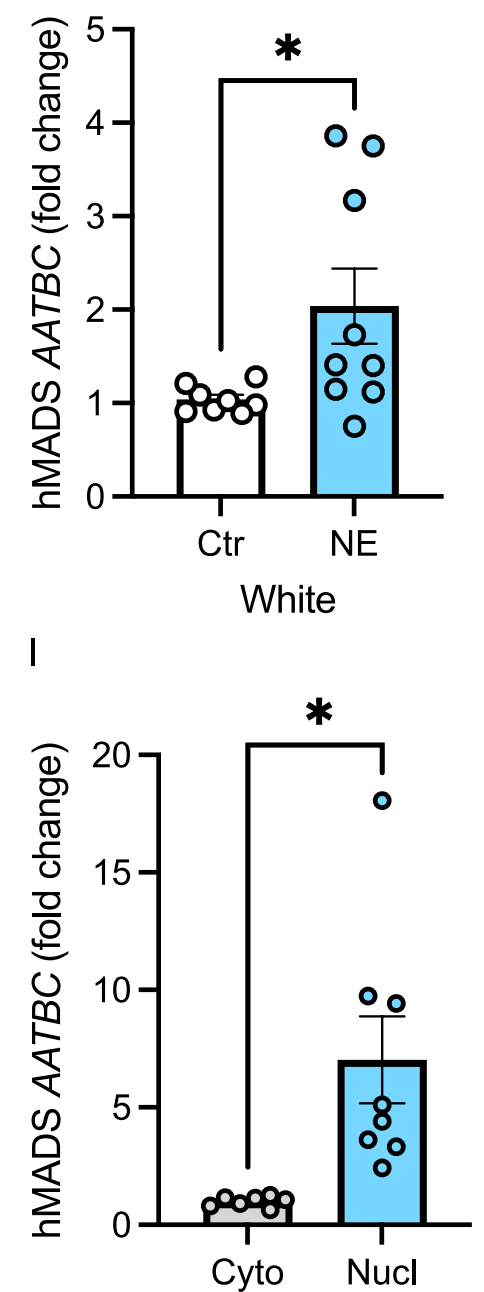

L

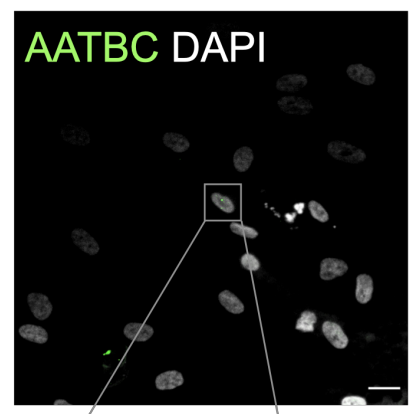

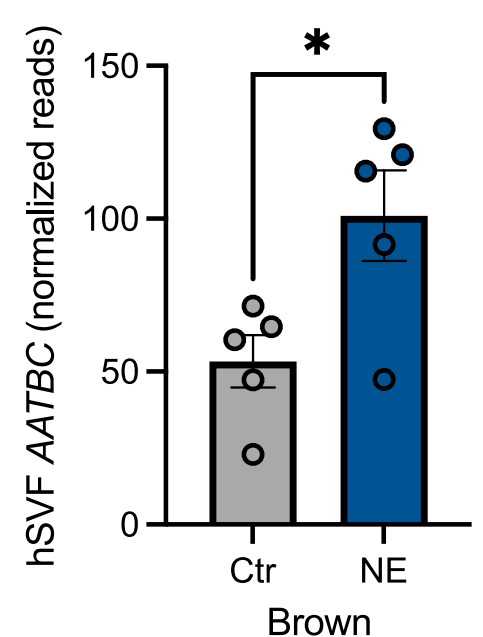

F

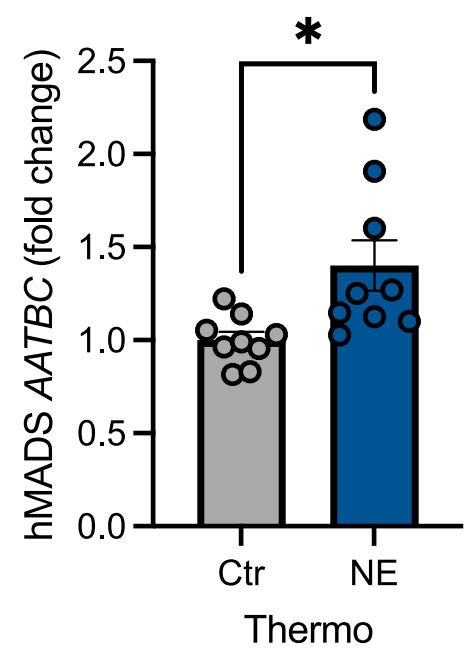

J

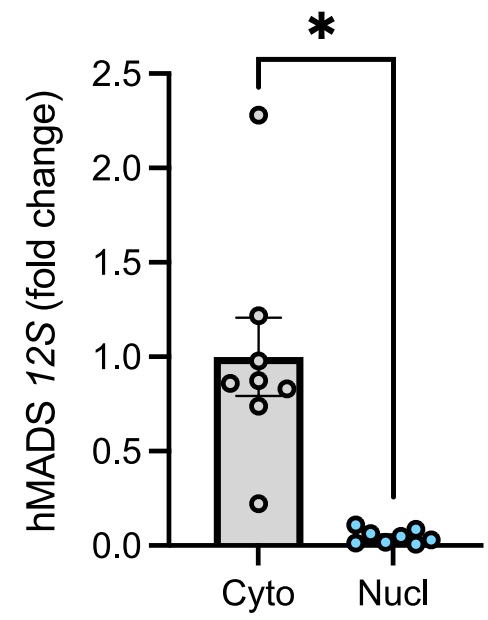

M
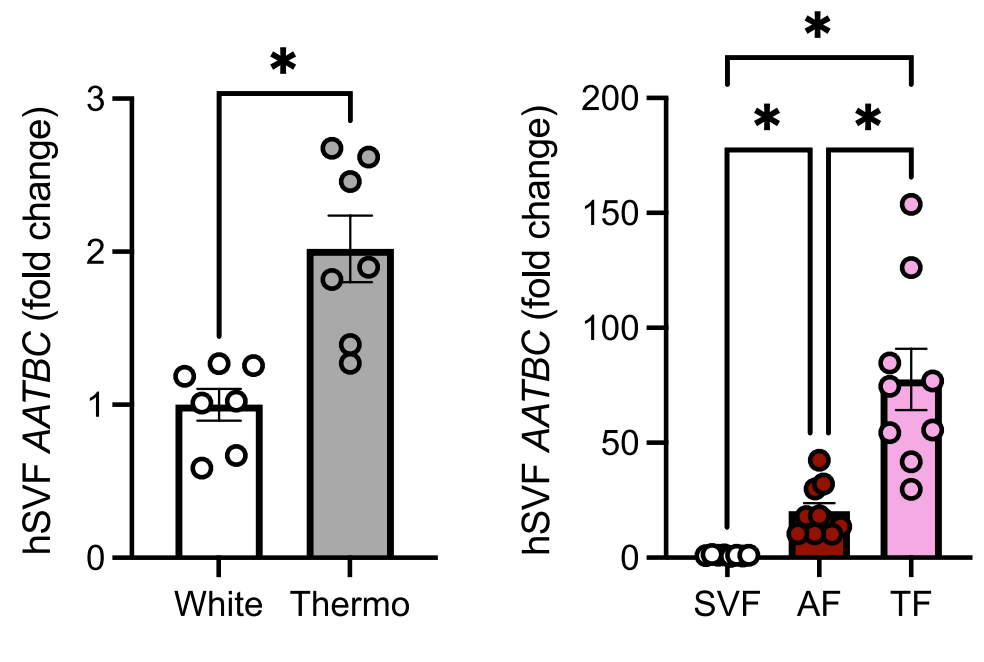

G

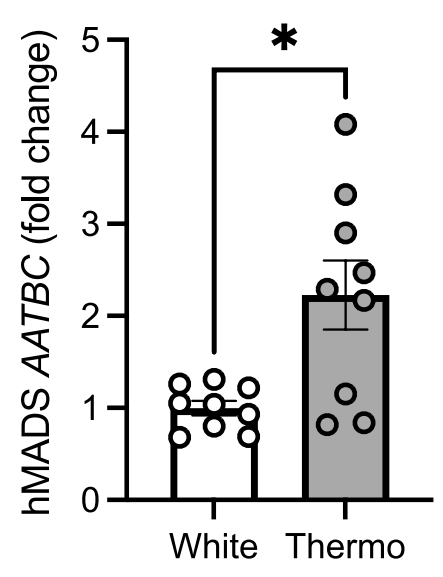

$\mathrm{H}$

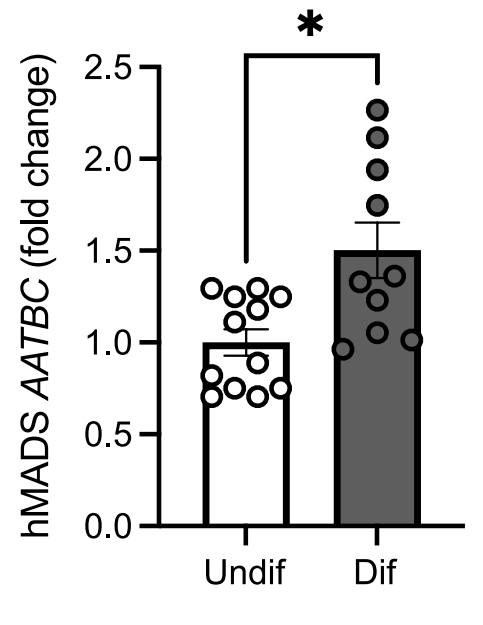

$\mathrm{K}$

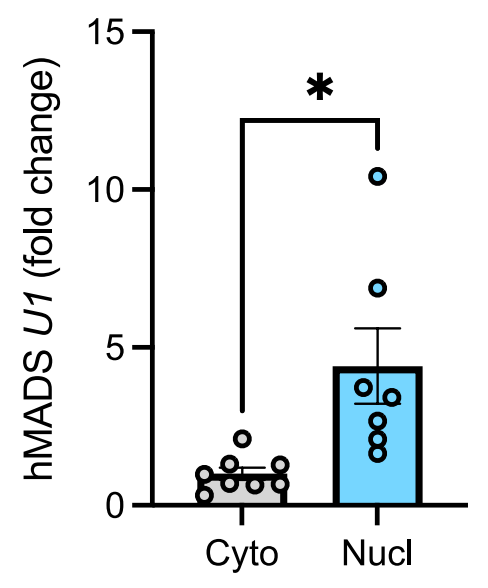

AV_AATBC

\section{AATBC DAPI}

霖
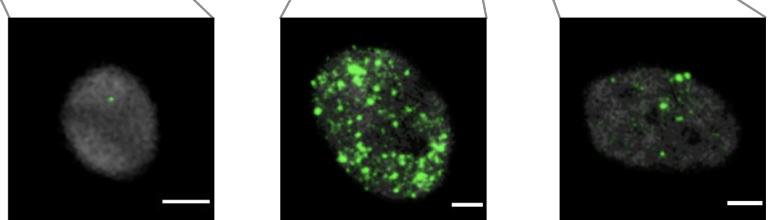

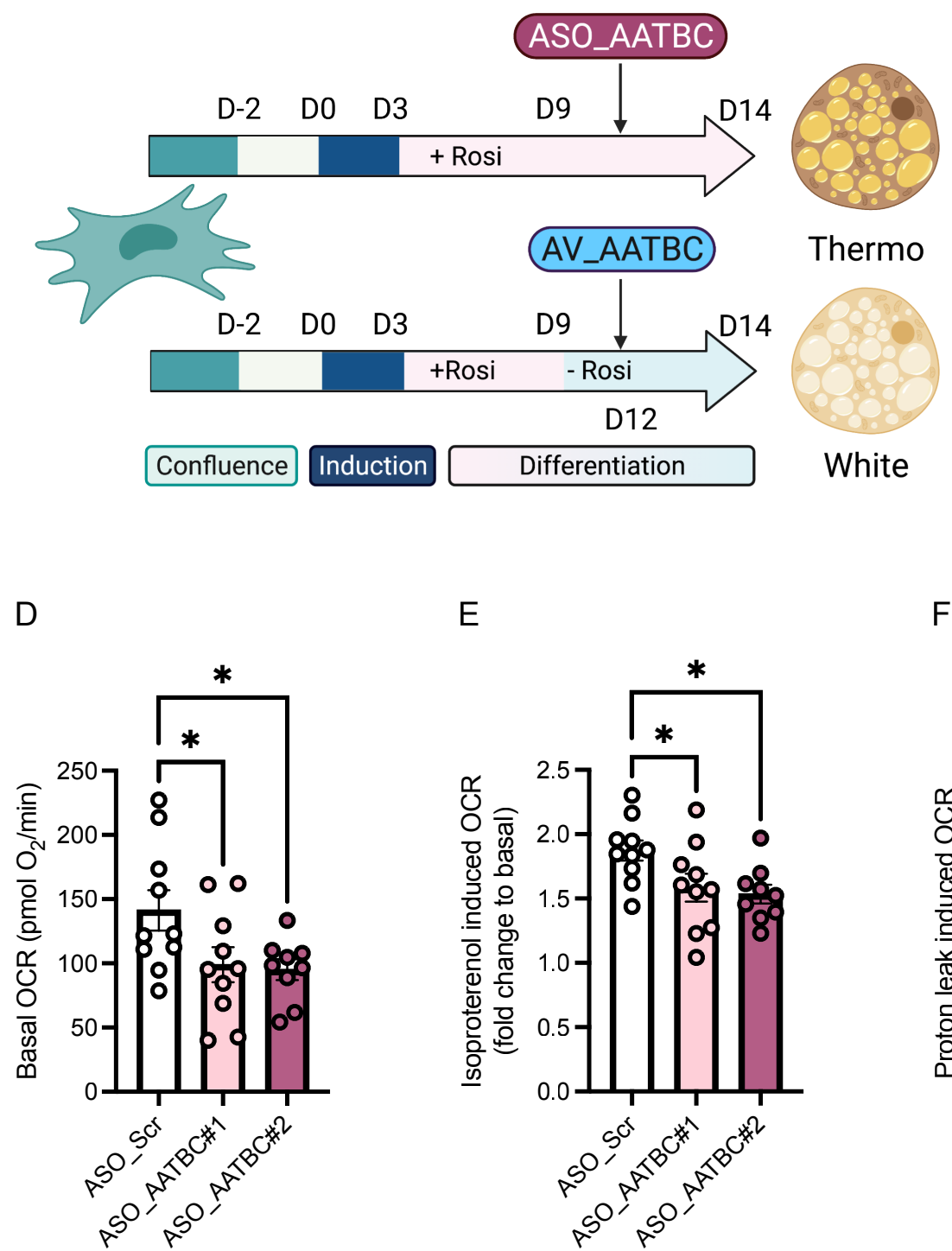

$\mathrm{H}$
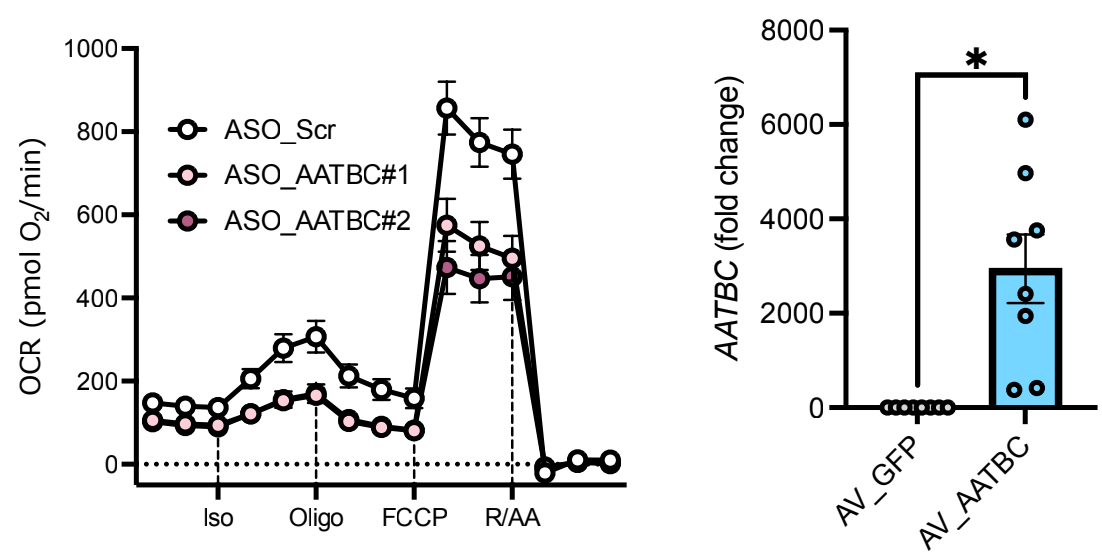

$\mathrm{N}$

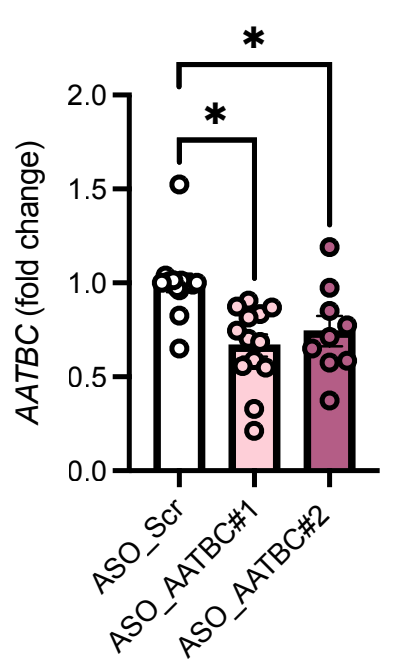

$\mathrm{F}$

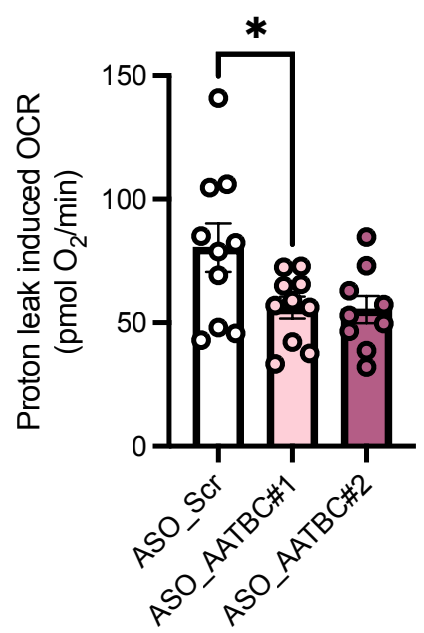

$J$

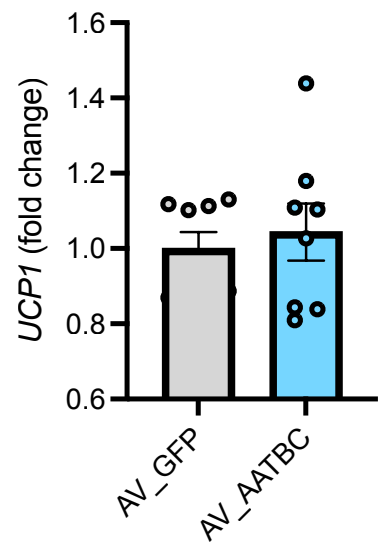

$\mathrm{O}$

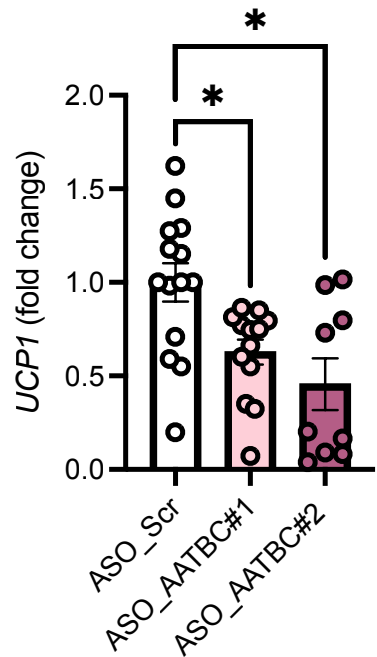

G
$\mathrm{K}$
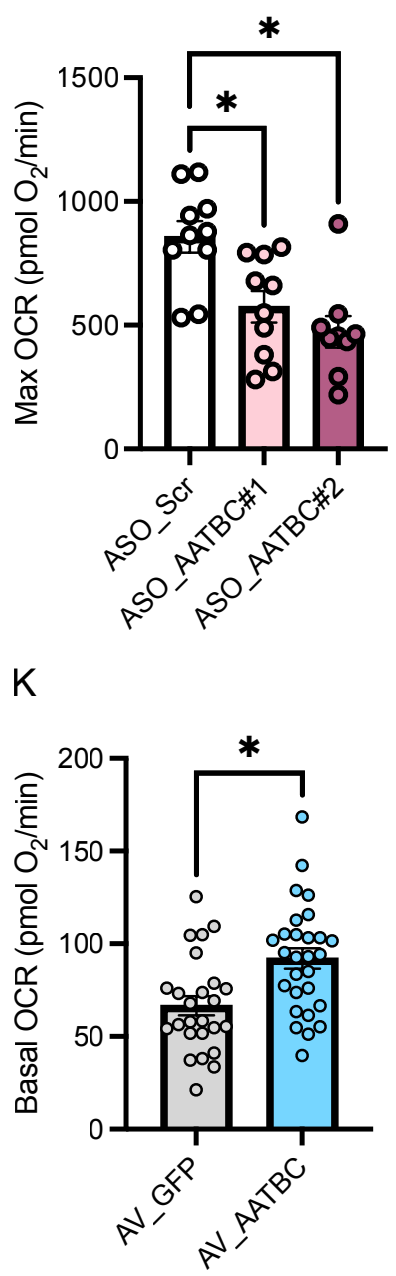

K
L

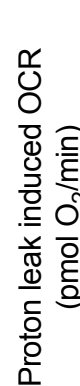

M

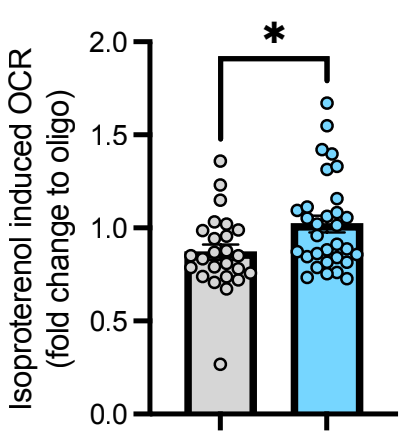

त्र
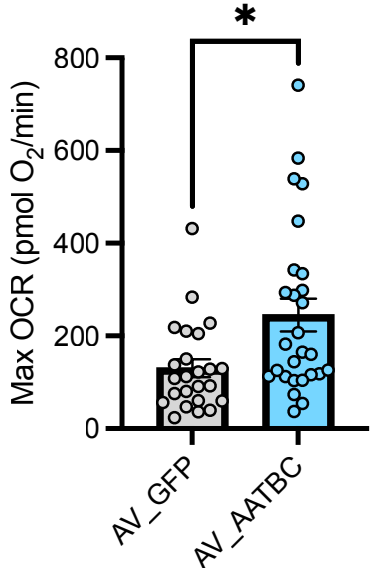

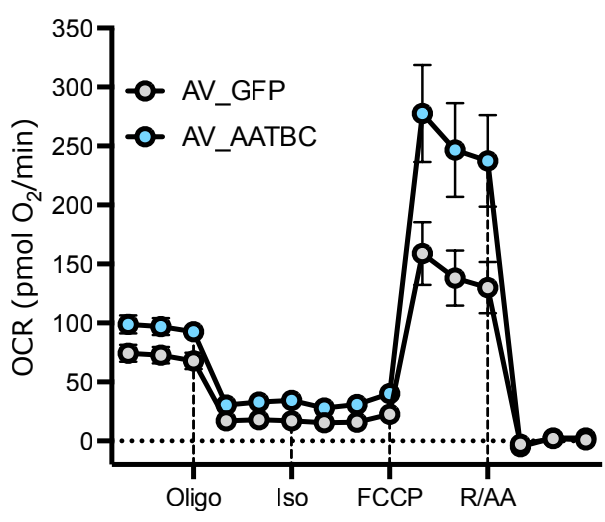


bioRxiv preprint doi: https://doi.org/10.1101/2021.08.09.455681; this version posted August 9, 2021. The copyright holder for this preprint Figure 4 (which was not certified by peer review) iBthe author/funder. All rights reservedCNo reuse allowed without permissiळD.

Original

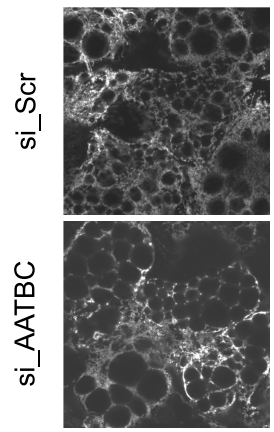

E

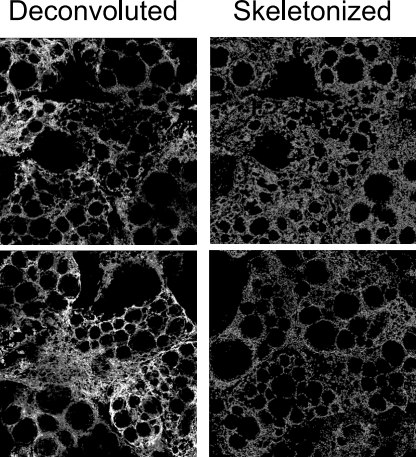

F

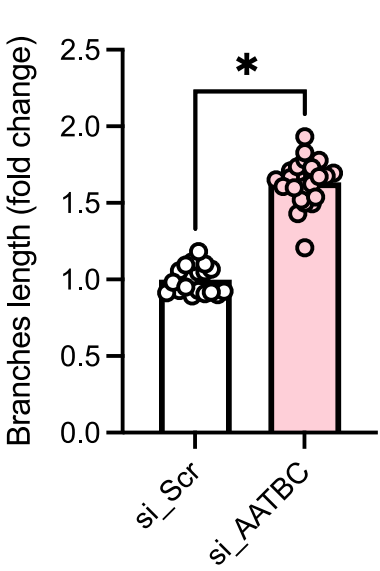

G

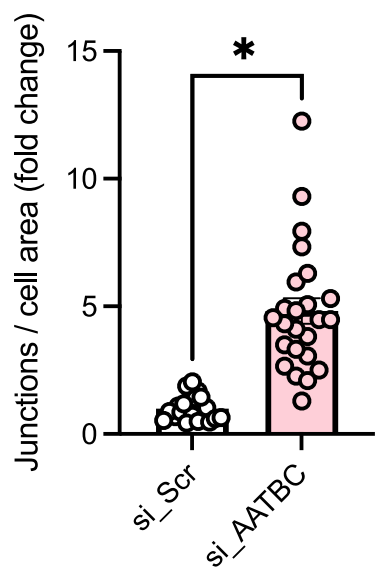

$\mathrm{H}$

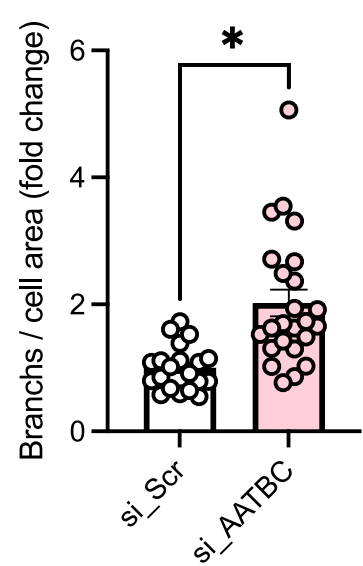

si_Scr si_AATBC
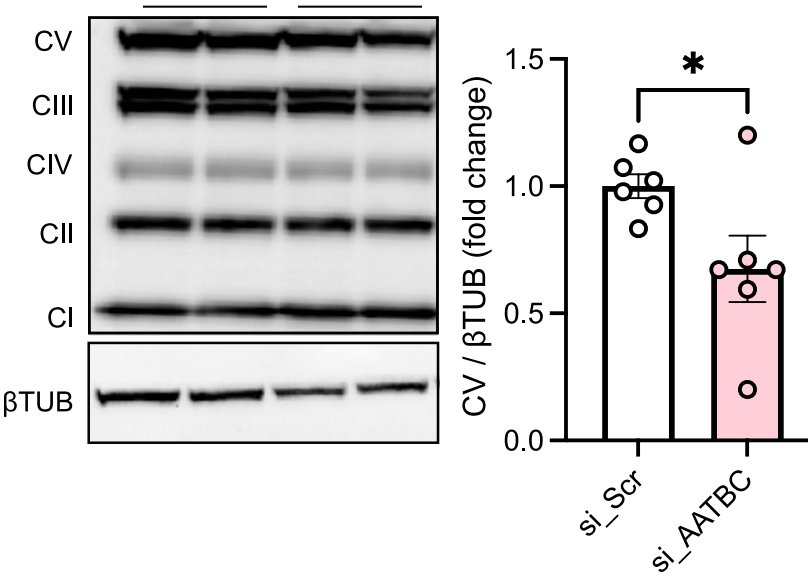

$\mathrm{K}$

$\mathrm{L}$
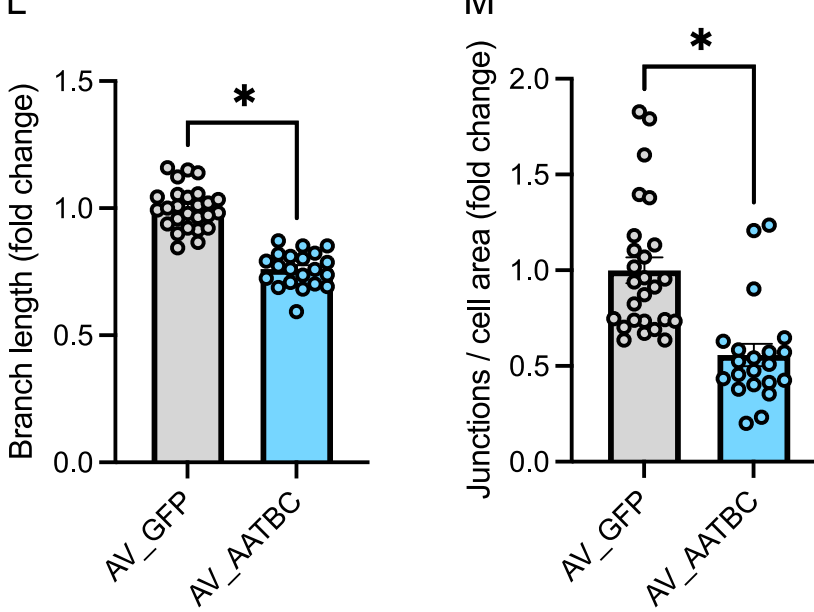

$\mathrm{R}$
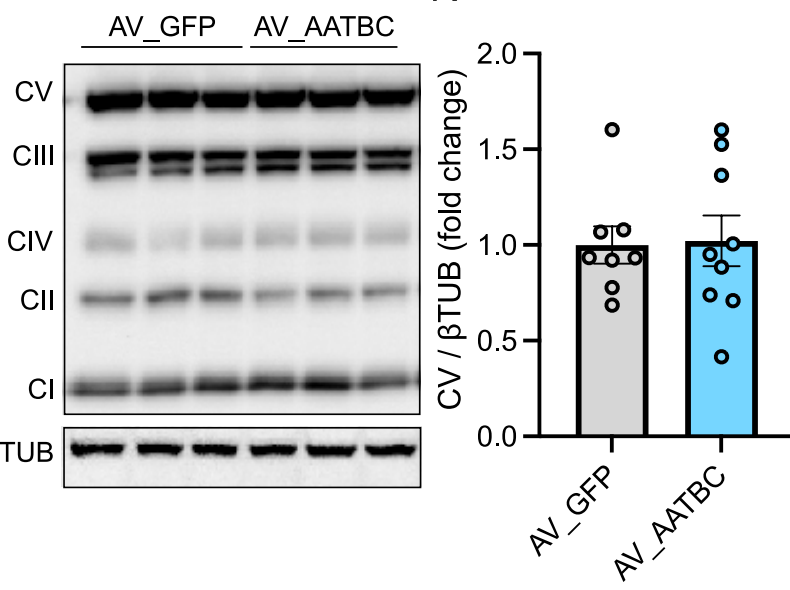

0

P

\section{(1)}
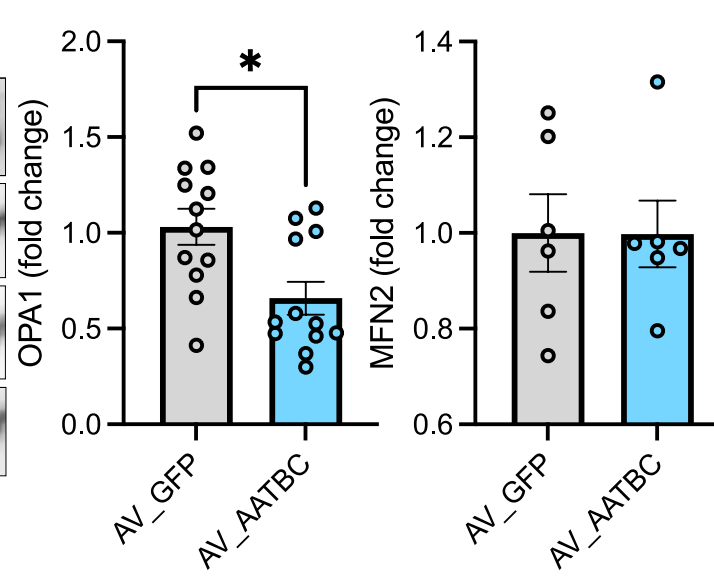

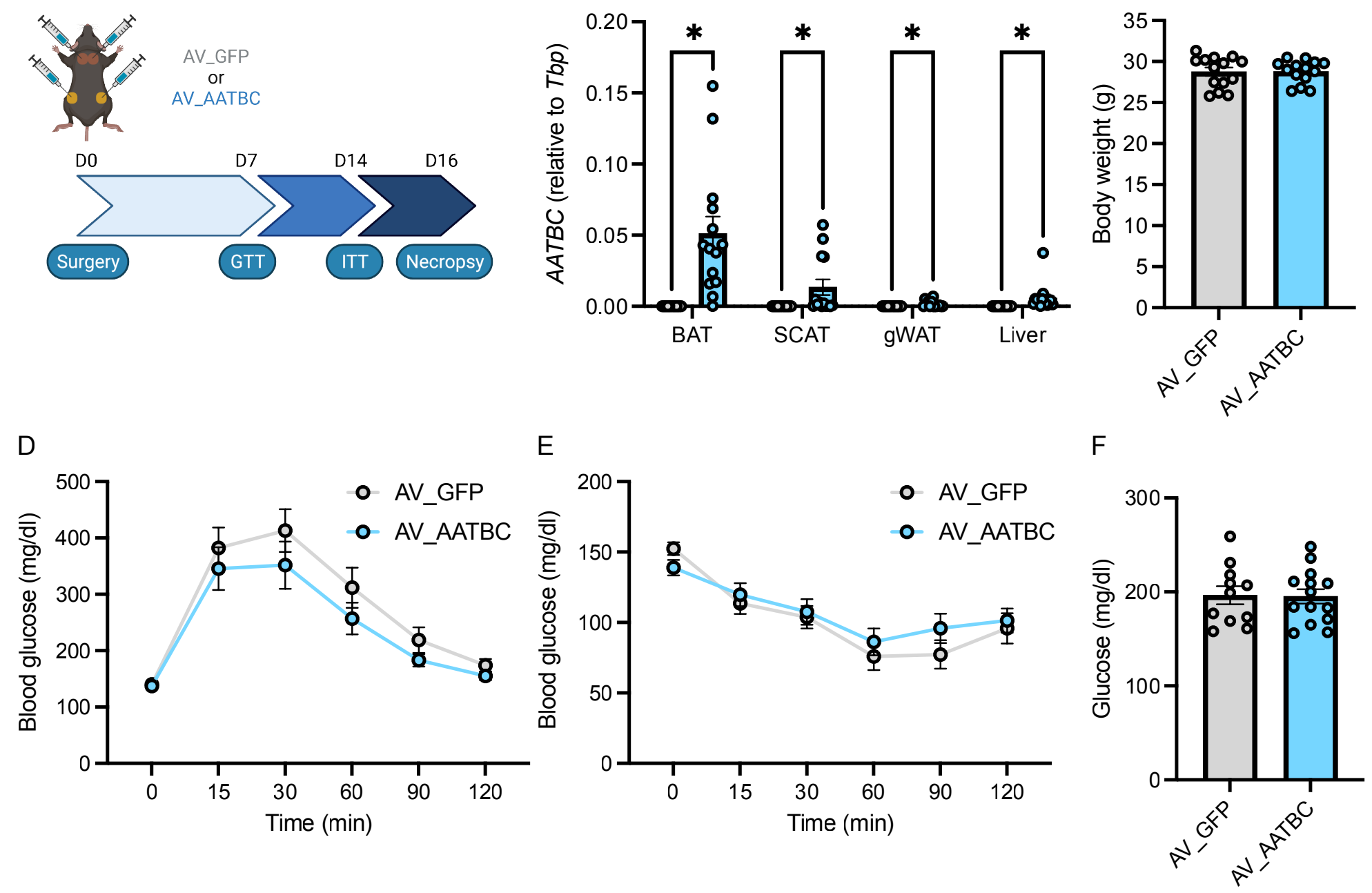

G

$\mathrm{H}$
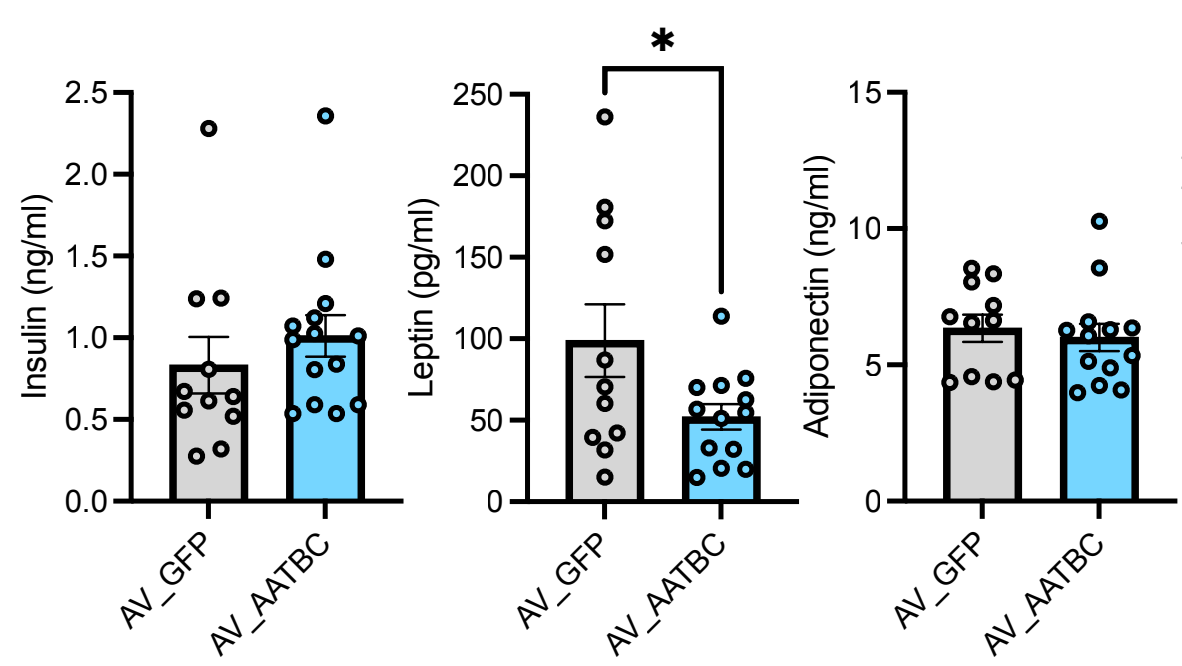

L

M

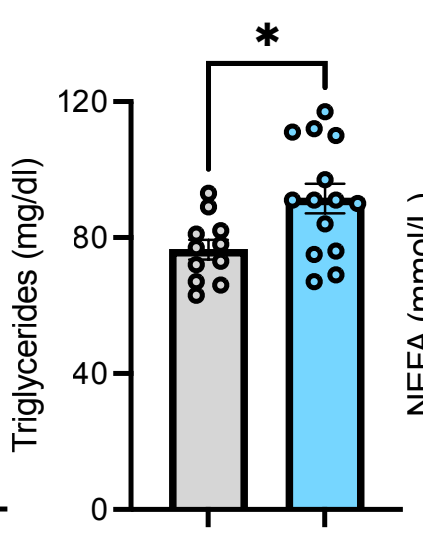

K
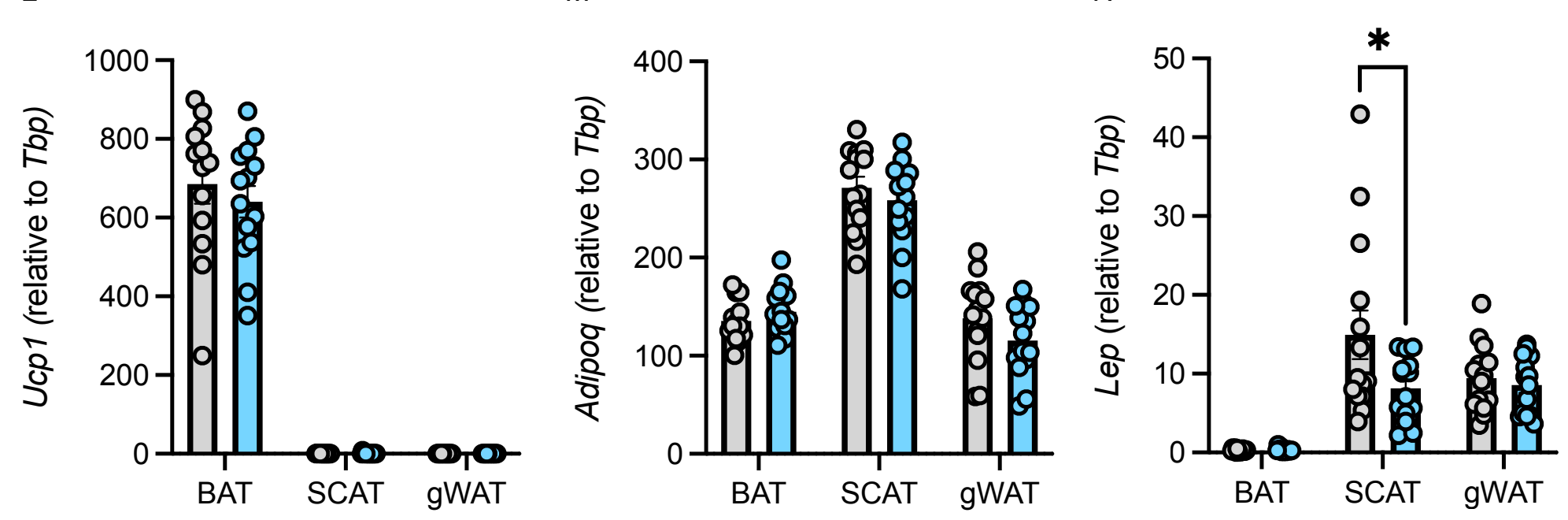
bioRxiv preprint doi: https://doi.org/10.1101/2021.08.09.455681; this version posted August 9, 2021. The copyright holder for this prepript (which was not certified by peer review) is the author/funder. All rights reserved. No reuse allowed without permission.

A

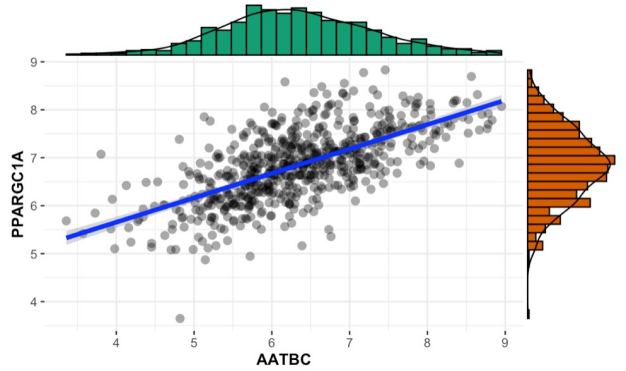

visWAT Females
B

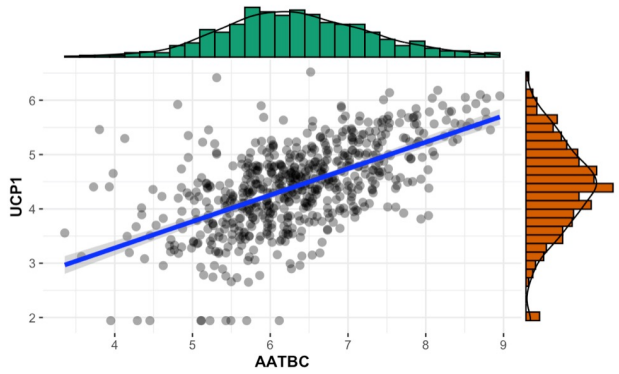

visWAT Females

E

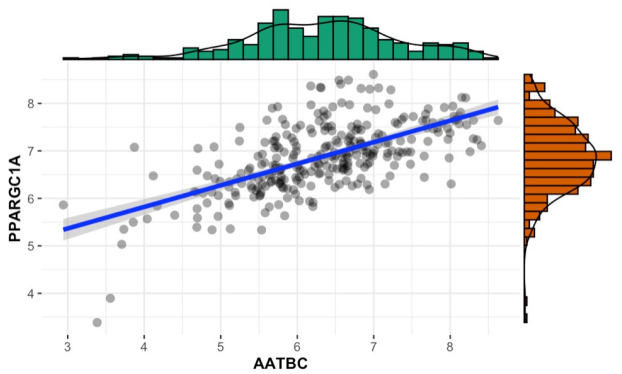

visWAT Males

G

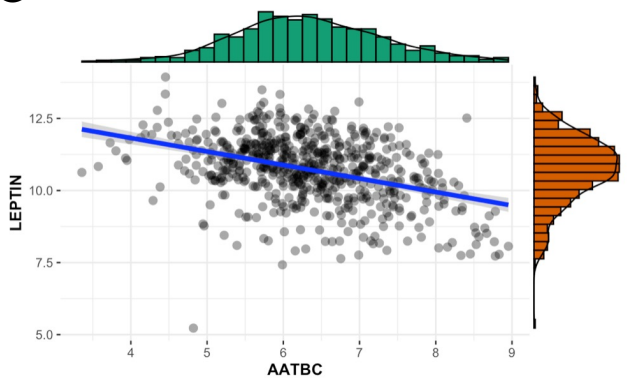

visWAT Females

$J$

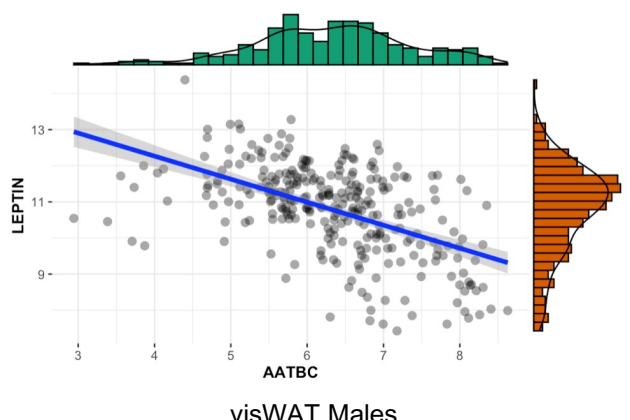

visWAT Males

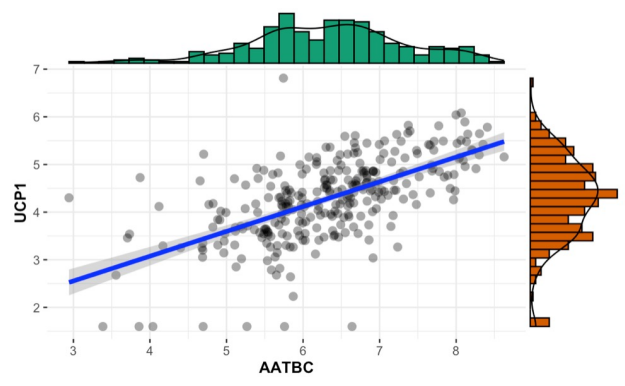

visWAT Males

$\mathrm{H}$

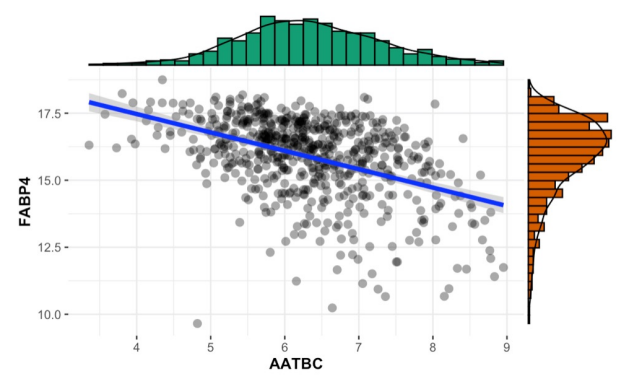

visWAT Females

K

visWAT Males
C

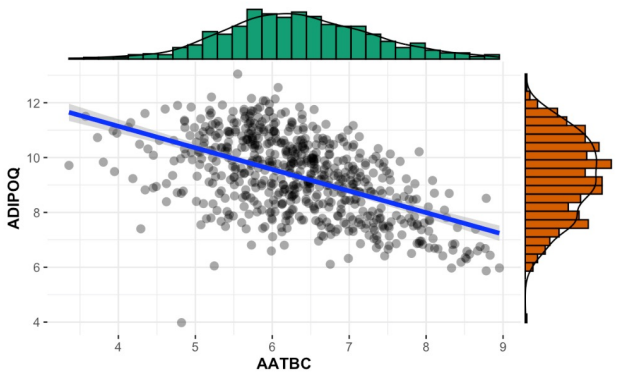

visWAT Females

F

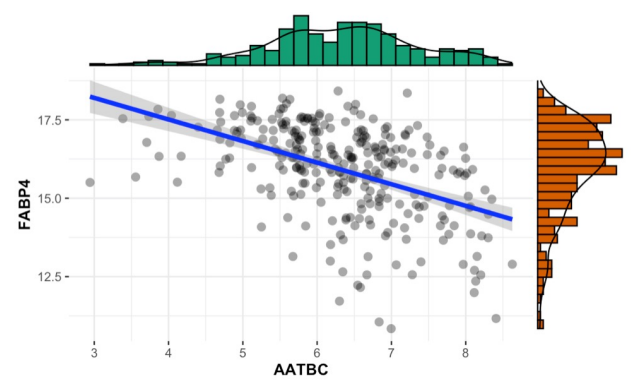

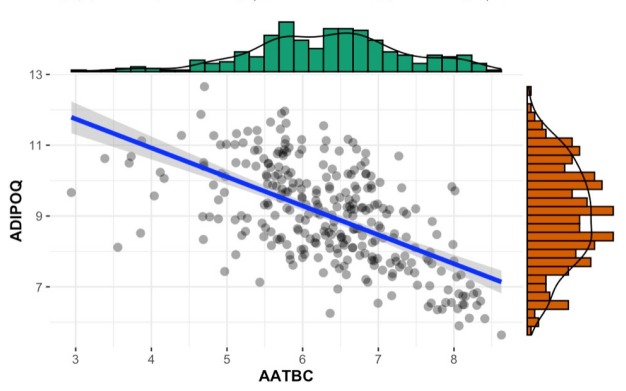

visWAT Males

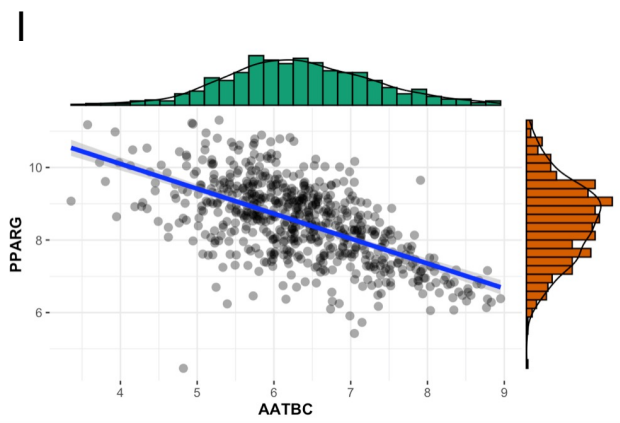

visWAT Females

L

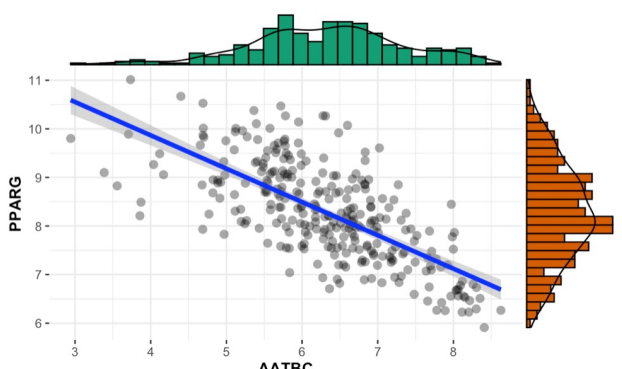

visWAT Males 